\title{
Loop Quantum Gravity
}

\author{
Carlo Rovelli \\ Department of Physics and Astronomy \\ University of Pittsburgh, Pittsburgh PA 15260, USA \\ rovelli@pitt.edu \\ http://www.pitt.edu/ ${ }^{\sim}$ rovelli \\ Published on 26 January 1998 \\ www.livingreviews.org/Articles/Volume1/1998-1rovelli \\ Living Reviews in Relativity \\ Published by the Max-Planck-Institute for Gravitational Physics \\ Albert Einstein Institute, Potsdam, Germany
}

\begin{abstract}
The problem of finding the quantum theory of the gravitational field, and thus understanding what is quantum spacetime, is still open. One of the most active of the current approaches is loop quantum gravity. Loop quantum gravity is a mathematically well-defined, non-perturbative and background independent quantization of general relativity, with its conventional matter couplings. Research in loop quantum gravity today forms a vast area, ranging from mathematical foundations to physical applications. Among the most significant results obtained are: (i) The computation of the physical spectra of geometrical quantities such as area and volume, which yields quantitative predictions on Planck-scale physics. (ii) A derivation of the Bekenstein-Hawking black hole entropy formula. (iii) An intriguing physical picture of the microstructure of quantum physical space, characterized by a polymer-like Planck scale discreteness. This discreteness emerges naturally from the quantum theory and provides a mathematically well-defined realization of Wheeler's intuition of a spacetime "foam". Long standing open problems within the approach (lack of a scalar product, over-completeness of the loop basis, implementation of reality conditions) have been fully solved. The weak part of the approach is the treatment of the dynamics: at present there exist several proposals, which are intensely debated. Here, I provide a general overview of ideas, techniques, results and open problems of this candidate theory of quantum gravity, and a guide to the relevant literature.
\end{abstract}

(c)1998 Max-Planck-Gesellschaft and the authors. Further information on copyright is given at http://www.livingreviews.org/Info/Copyright/. For permission to reproduce the article please contact livrev@aei-potsdam.mpg.de. 


\section{Article Amendments}

On author request a Living Reviews article can be amended to include errata and small additions to ensure that the most accurate and up-to-date information possible is provided. For detailed documentation of amendments, please go to the article's online version at

http://www .livingreviews .org/Articles/Volume1/1998-1rovelli/.

Owing to the fact that a Living Reviews article can evolve over time, we recommend to cite the article as follows:

Rovelli, C., "Loop Quantum Gravity",

Living Rev. Relativity, 1, (1998), 1. [Online Article]: cited on <date>, http://www.livingreviews.org/Articles/Volume1/1998-1rovelli/.

The date in 'cited on $<$ date $>$ ' then uniquely identifies the version of the article you are referring to. 


\section{Contents}

1. Introduction 4

2 Quantum Gravity: Where are We? 6

2.1 What is the problem? The view of a high energy physicist . . . 6

2.2 What is the problem? The view of a relativist . . . . . . . . 7

2.3 Strings or loopst $\ldots \ldots \ldots \ldots \ldots \ldots \ldots$

3 History of Loop Quantum Gravity, Main Steps 10

$\begin{array}{ll}4 \text { Resources } & 15\end{array}$

\begin{tabular}{|lll}
5 & Main Ideas and Physical Inputs & 17
\end{tabular}

5.1 Quantum field theory on a differentiable manifold . . . . . . . . . 17

5.2 One additional assumption $\ldots \ldots \ldots \ldots \ldots$

5.3 Physical meaning of diffeomorphism invariance, and its implementation in the quantum theory . . . . . . . . . . . . . . . 19

5.4 Problems not addressed $\ldots \ldots \ldots \ldots$. . . . . . . . . 20

6 The Formalism 22

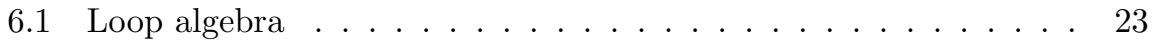

6.2 Loop quantum gravity . . . . . . . . . . . . . . . . . . . . 24

6.3 Loop states and spin network states $\ldots \ldots \ldots \ldots$

6.4 Relation between spin network states and loop states and diagrammatic representation of the states . . . . . . . . . . . 28

6.5 The representation $\ldots \ldots \ldots \ldots \ldots \ldots$

6.6 Algebraic version ("loop representation") and differential version ("connection representation") of the formalism, and their equiv-

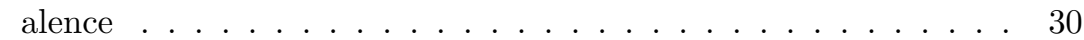

6.7 Other structures in $\mathbb{H} \ldots \ldots \ldots \ldots \ldots \ldots \ldots$

6.8 Diffeomorphism invariance $\ldots \ldots \ldots \ldots \ldots$

6.9 Dynamics $\ldots \ldots \ldots \ldots \ldots \ldots \ldots$

6.10 Unfreezing the frozen time formalism: the covariant form of loop

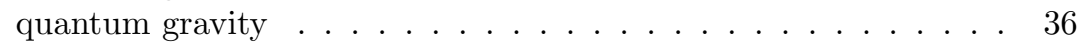

7 Physical Results $\quad 40$

T.T Technical . . . . . . . . . . . . . . . . 40

7.2 Physical . . . . . . . . . . . . . . . . . 41

8 Main Open Problems and Main Current Lines of Investigation 47

9 Short Summary and Conclusion $\quad 52$

9. Conclusion . . . . . . . . . . . . . . . . 52

10 Acknowledgments 54

$\begin{array}{ll}\text { References } & 55\end{array}$

Living Reviews in Relativity (1998-1)

http://www.livingreviews.org 


\section{Introduction}

The loop approach to quantum gravity is ten years old. The first announcement of this approach was given at a conference in India in 1987 [182]. This tenth anniversary is a good opportunity to attempt an assessment of what has and what has not been accomplished in these ten years of research and enthusiasm.

During these ten years, loop quantum gravity has grown into a wide research area and into a solid and rather well-defined tentative theory of the quantum gravitational field. The approach provides a candidate theory of quantum gravity. It provides a physical picture of Planck scale quantum geometry, calculation techniques, definite quantitative predictions, and a tool for discussing classical problems such as black hole thermodynamics.

We do not know whether this theory is physically correct or not. Direct or indirect experimental corroboration of the theory is lacking. This is the case, unfortunately, for all present approaches to quantum gravity, due, of course, to the minuteness of the scale at which quantum properties of spacetime (presumably) manifest themselves. In the absence of direct experimental guidance, we can evaluate a theory and compare it with alternative theories only in terms of internal consistency and consistency with what we do know about Nature.

Long standing open problems within the theory (such as the lack of a scalar product, the incompleteness of the loop basis and the related difficulty of dealing with identities between states, or the difficulty of implementing the reality conditions in the quantum theory) have been solidly and satisfactorily solved. But while it is fairly well developed, loop quantum gravity is not yet a complete theory. Nor has its consistency with classical general relativity been firmly established yet. The sector of the theory which has not yet solidified is the dynamics, which exists in several variants presently under intense scrutiny. On the other hand, in my opinion the strength of the theory is its compelling capacity to describe quantum spacetime in a background independent nonperturbative manner, and its genuine attempt to synthesize the conceptual novelties introduced by quantum mechanics with the ones introduced by general relativity.

The other large research program for a quantum theory of gravity, besides loop quantum gravity, is string theory, which is a tentative theory as well. String theory is more ambitious than loop gravity, since it also aims at unifying all known fundamental physics into a single theory. In section 2.3], I will compare strengths and weaknesses of these two competing approaches to quantum gravity.

This "living review" is intended to be a tool for orienting the reader to the field of loop gravity. Here is the plan for the review:

- Section 2, "Quantum Gravity: Where are We?", is an introduction to the problem, the reason of its relevance, and the present state of our knowledge.

- Section 3, "History of Loop Quantum Gravity", is a short overview of the historical development of the theory.

Living Reviews in Relativity (1998-1)

http://www.livingreviews.org 


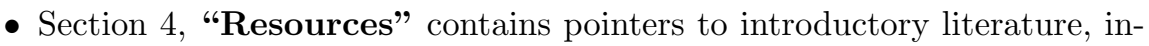
stitutions where loop gravity is studied, web pages, and other information that may be of use to students and researchers.

- Section 5, "Main Ideas and Physical Inputs", discusses, at a rather technical level, the physical and mathematical ideas on which loop quantum gravity is based.

- The actual theory is described in detail in section 6, "The Formalism".

- Section 7, "Results", is devoted to the results that have been derived from the theory. I have divided results in two groups. First, the "technical" results (7.1), namely the ones that are important for the construction and the understanding of the theory itself, or that warrant the theory's consistency. Second, the "physical" results (7.2): that is, what the theory says about the physical world.

- In section 8, "Open Problems and Current Lines of Investigation", I illustrate what I consider to be the main open problems and currently active lines of research.

- In section 9, "Short Summary and Conclusion", I summarize very briefly the state and the results of the theory, and present (necessarily very preliminary!) conclusions.

At the cost of several repetitions, the structure of this review is very modular: Sections are to a large extent independent of each other, have different styles, and can be combined according to the interest of the reader. A reader interested only in a very brief overview of the theory and its results can find this in section 9. Graduate students or persons of general culture may get a general idea of what goes on in this field and its main ideas from sections 2 and 7 . If interested only in the technical aspects of the theory and its physical results, one can read sections 6 and 7 alone. Scientists working in this field can use sections 6 and 7 as a reference, and I hope they will find sections 2, 3, 5 and 8 stimulating.

I will not enter into technical details. However, I will point to the literature where these details are discussed. I have tried to be as complete as possible in indicating all relevant aspects and potential difficulties of the issues discussed.

The literature in this field is vast, and I am sure that there are works whose existence or relevance I have failed to recognize. I sincerely apologize to the authors whose contributions I have neglected or under-emphasized, and I strongly urge them to contact me to help me make this review more complete. The "living reviews" are constantly updated, and I will be able to correct errors and omissions in the future.

Living Reviews in Relativity (1998-1) http://www.livingreviews.org 


\section{Quantum Gravity: Where are We?}

This is a non-technical section in which I illustrate the problem of quantum gravity in general, its origin, its importance, and the present state of our knowledge in this regard.

The problem of describing the quantum regime of the gravitational field is still open. There are tentative theories, and competing research directions. For an overview, see [120]. The two largest research programs are string theory and loop quantum gravity. But several other directions are being explored, such as twistor theory [15.3], noncommutative geometry [68], simplicial quantum gravity $[0,66,62$, I] , Euclidean quantum gravity [103, [106], the Null Surface Formulation $[85,86,87]$ and others.

String theory and loop quantum gravity differ not only because they explore distinct physical hypotheses, but also because they are expressions of two separate communities of scientists, scientists who have sharply distinct prejudices, and who view the problem of quantum gravity in surprisingly different manners.

\subsection{What is the problem? The view of a high energy physicist}

High energy physics has obtained spectacular successes during this century, culminating with the (far from linear) establishment of quantum field theory as the general form of dynamics and with the comprehensive success of the $S U(3) \times S U(2) \times U(1)$ Standard Model. Thanks to this success, now a few decades old, physics is in a position in which it has very rarely been: There are no experimental results that clearly challenge, or clearly escape, the present fundamental theory of the world. The theory we have encompasses virtually everything - except gravitational phenomena. From the point of view of a particle physicist, gravity is then simply the last and weakest of the interactions. It is natural to try to understand its quantum properties using the strategy that has been so successful for the rest of microphysics, or variants of this strategy. The search for a conventional quantum field theory capable of embracing gravity has spanned several decades and, through an adventurous sequence of twists, moments of excitement and disappointments, has lead to string theory. The foundations of string theory are not yet well understood; and it is not yet entirely clear how a supersymmetric theory in 10 or 11 dimensions can be concretely used for deriving comprehensive univocal predictions about our world. 1 But string theory may claim extremely remarkable theoretical successes and is today the leading and most widely investigated candidate theory of quantum gravity.

In string theory, gravity is just one of the excitations of a string (or other

\footnotetext{
${ }^{1}$ I have heard the following criticism of loop quantum gravity: "Loop quantum gravity is certainly physically wrong, because: (1) It is not supersymmetric, and (2) it is formulated in four dimensions". But experimentally, the world still insists on looking four-dimensional and not supersymmetric. In my opinion, people should be careful to not be blinded by their own speculation and inadvertently mistake interesting hypotheses (such as supersymmetry and high-dimensions) for established truths.
}

Living Reviews in Relativity (1998-1)

http://www.livingreviews.org 
Loop Quantum Gravity

extended object) living over some background metric space. The existence of such background metric space, over which the theory is defined, is needed for the formulation and for the interpretation of the theory. This is the case not only in perturbative string theory, but, to my understanding, in the recent attempts at a non-perturbative definition of the theory, such as $M$ theory. Thus, for a physicist with a high energy background, the problem of quantum gravity is now reduced to an aspect of the problem of understanding: What is the mysterious nonperturbative theory that has perturbative string theory as its perturbation expansion? And how does one extract information on Planck scale physics from it?

\subsection{What is the problem? The view of a relativist}

For a relativist, on the other hand, the idea of a fundamental description of gravity in terms of physical excitations over a background metric space sounds physically very wrong. The key lesson learned from general relativity is that there is no background metric over which physics happens (unless, of course, in approximations). The world is more complicated than that. Indeed, for a relativist, general relativity is much more than the field theory of a particular force. Rather, it is the discovery that certain classical notions about space and time are inadequate at the fundamental level; they require modifications which are possibly as basic as the ones that quantum mechanics introduced. One of these inadequate notions is precisely the notion of a background metric space, (flat or curved), over which physics happens. This profound conceptual shift has led to the understanding of relativistic gravity, to the discovery of black holes, to relativistic astrophysics and to modern cosmology.

From Newton to the beginning of this century, physics has had a solid foundation in a small number of key notions such as space, time, causality and matter. In spite of substantial evolution, these notions remained rather stable and self-consistent. In the first quarter of this century, quantum theory and general relativity have deeply modified this foundation. The two theories have obtained solid success and vast experimental corroboration, and can now be considered established knowledge. Each of the two theories modifies the conceptual foundation of classical physics in an (more or less) internally consistent manner, but we do not have a novel conceptual foundation capable of supporting both theories. This is why we do not yet have a theory capable of predicting what happens in the physical regime in which both theories are relevant, the regime of Planck scale phenomena, $10^{-33} \mathrm{~cm}$.

General relativity has taught us not only that space and time share the property of being dynamical with the rest of the physical entities, but also -more crucially - that spacetime location is relational only (see section 5.3). Quantum mechanics has taught us that any dynamical entity is subject to Heisenberg's uncertainty at small scale. Therefore, we need a relational notion of a quantum spacetime in order to understand Planck scale physics.

Thus, for a relativist, the problem of quantum gravity is the problem of bringing a vast conceptual revolution, begun with quantum mechanics and with

Living Reviews in Relativity (1998-1)

http://www.livingreviews.org 
general relativity, to a conclusion and to a new synthesis. 2 In this synthesis the notions of space and time need to be deeply reshaped in order to take into account what we have learned with both our present "fundamental" theories.

Unlike perturbative or nonperturbative string theory, loop quantum gravity is formulated without a background spacetime. Loop quantum gravity is thus a genuine attempt to grasp what quantum spacetime is at the fundamental level. Accordingly, the notion of spacetime that emerges from the theory is profoundly different from the one on which conventional quantum field theory or string theory is based.

\subsection{Strings or loops?}

Above I have emphasized the radically distinct cultural paths leading to string theory and loop quantum gravity. Here I attempt to compare the actual achievements the two theories have obtained so far regarding the description of Planck scale physics.

Once more, however, I want to emphasize that, whatever prejudices this or that physicist may have, both theories are tentative: As far as we really know, either, or both, theories could very well turn out to be physically entirely wrong. And I do not mean that they could be superseded: I mean that all their specific predictions could be disproved by experiments. Nature does not always share our aesthetic judgments, and the history of theoretical physics is full of enthusiasm for strange theories turned into disappointment. The arbiters in science are experiments, and not a single experimental result supports, not even very indirectly, any of the current theories that go beyond the Standard Model and general relativity. To the contrary, all the predictions made so far by theories that go beyond the Standard Model and general relativity (proton decay, supersymmetric particles, exotic particles, solar system dynamics) have for the moment been punctually falsified by experiments. Comparing this situation with the astonishing experimental success of the Standard Model and classical general relativity should make us very cautious, I believe. Lacking experiments, theories can only be compared on completeness and aesthetic criteria - criteria, one should not forget, that according to many favored Ptolemy over Copernicus at some point.

The main merits of string theory are that it provides a superbly elegant unification of known fundamental physics, and that it has a well defined perturbation expansion, finite order by order. Its main incompletenesses are that its non-perturbative regime is poorly understood, and that we do not have a background-independent formulation of the theory. In a sense, we do not really know what the theory we are talking about is. Because of this poor understanding of the non perturbative regime of the theory, Planck scale physics and genuine quantum gravitational phenomena are not easily controlled: Except for a few computations, there has not been much Planck scale physics derived from string theory so far. There are, however, two sets of remarkable physical results.

${ }^{2}$ For a detailed discussion of this idea, see [Iㅈ] ] and [[197].

Living Reviews in Relativity (1998-1)

http://www.livingreviews.org 
Loop Quantum Gravity

The first is given by some very high energy scattering amplitudes that have been computed (see for instance [3, 4, 5, 6, 20.9, 200]). An intriguing aspect of these results is that they indirectly suggest that geometry below the Planck scale cannot be probed -and thus in a sense does not exist- in string theory. The second physical achievement of string theory (which followed the d-branes revolution) is the recent derivation of the Bekenstein-Hawking black hole entropy formula for certain kinds of black holes [1.98, 피, ए10.9, 108].

The main merit of loop quantum gravity, on the other hand, is that it provides a well-defined and mathematically rigorous formulation of a backgroundindependent, non-perturbative generally covariant quantum field theory. The theory provides a physical picture and quantitative predictions of the world at the Planck scale. The main incompleteness of the theory is regarding the dynamics, formulated in several variants. So far, the theory has lead to two main sets of physical results. The first is the derivation of the (Planck scale) eigenvalues of geometrical quantities such as areas and volumes. The second is the derivation of black hole entropy for "normal" black holes (but only up to the precise numerical factor).

Finally, strings and loop gravity may not necessarily be competing theories: There might be a sort of complementarity, at least methodological, between the two. This is due to the fact that the open problems of string theory are with respect to its background-independent formulation, and loop quantum gravity is precisely a set of techniques for dealing non-perturbatively with background independent theories. Perhaps the two approaches might even, to some extent, converge. Undoubtedly, there are similarities between the two theories: first of all the obvious fact that both theories start with the idea that the relevant excitations at the Planck scale are one dimensional objects - call them loops or strings. I understand that in another living review to be published in this journal Lee Smolin explores the possible relations between string theory and loop gravity [1.91].

Living Reviews in Relativity (1998-1) http://www.livingreviews.org 


\section{History of Loop Quantum Gravity, Main Steps}

The following chronology does not exhaust the literature on loop quantum gravity. It only indicates the key steps in the construction of the theory, and the first derivation of the main results. For more complete references, see the following sections. (Due to the attempt to group similar results, some things may appear a bit out of the chronological order.)

\section{Connection formulation of classical general relativity Sen, Ashtekar.}

Loop quantum gravity is based on the formulation of classical general relativity, which goes under the name of "new variables", or "Ashtekar variables", or "connectio-dynamics" (in contrast to Wheeler's "geometrodynamics"). In this formulation, the field variable is a self-dual connection, instead of the metric, and the canonical constraints are simpler than in the old metric formulation. The idea of using a self-dual connection as field variable and the simple constraints it yields were discovered by Amitaba Sen [1.90]. Abhay Ashtekar realized that in the $S U(2)$ extended phase space a self-dual connection and a densitized triad field form a canonical pair $[\boldsymbol{8}, 9]$ and set up the canonical formalism based on such pair, which is the Ashtekar formalism. Recent works on the loop representation are not based on the original Sen-Ashtekar connection, but on a real variant of it, whose use has been introduced into Lorentzian general relativity by Barbero [40, 41, 42, 43]].

\section{Wilson loop solutions of the hamiltonian constraint} Jacobson, Smolin.

Soon after the introduction of the classical Ashtekar variables, Ted Jacobson and Lee Smolin realized in [127] that the Wheeler-DeWitt equation, reformulated in terms of the new variables, admits a simple class of exact solutions: the traces of the holonomies of the Ashtekar connection around smooth non-selfintersecting loops. In other words: The Wilson loops of the Ashtekar connection solve the Wheeler-DeWitt equation if the loops are smooth and non self-intersecting.

\section{The Loop Representation}

\section{Rovelli, Smolin.}

The discovery of the Jacobson-Smolin Wilson loop solutions prompted Carlo Rovelli and Lee Smolin [182, 163, 183., 184] to "change basis in the Hilbert space of the theory", choosing the Wilson loops as the new basis states for quantum gravity. Quantum states can be represented in terms of their expansion on the loop basis, namely as functions on a space of loops. This idea is well known in the context of canonical lattice Yang-Mills theory [2I]], and its application to continuous Yang-Mills theory had been explored by Gambini and Trias [95, 96], who developed a continuous "loop representation" much before the Rovelli-Smolin one. The difficulties of the loop representation in the context of Yang-Mills theory are cured by

Living Reviews in Relativity (1998-1)

http://www.livingreviews.org 
the diffeomorphism invariance of GR (see section 6.8 for details). The loop representation was introduced by Rovelli and Smolin as a representation of a classical Poisson algebra of "loop observables". The relation to the connection representation was originally derived in the form of an integral transform (an infinite dimensional analog of a Fourier transform) from functionals of the connection to loop functionals. Several years later, this loop transform was shown to be mathematically rigorously defined [[13]. The immediate results of the loop representation were two: The diffeomorphism constraint was completely solved by knot states (loop functionals that depend only on the knotting of the loops), making earlier suggestions by Smolin on the role of knot theory in quantum gravity [195] concrete; and (suitable [184, 1966] extensions of) the knot states with support on non-selfintersecting loops were proven to be solutions of all quantum constraints, namely exact physical states of quantum gravity.

\section{8 - Exact states of quantum gravity} Husain, Brügmann, Pullin, Gambini, Kodama.

The investigation of exact solutions of the quantum constraint equations, and their relation to knot theory (in particular to the Jones polynomial and other knot invariants) started soon after the formulation of the theory and has continued since [11, 57, 58, 5.9, 60, 157, 92, 94, 1301, 8.9].

\section{9 - Model theories}

Ashtekar, Husain, Loll, Marolf, Rovelli, Samuel, Smolin, Lewandowski, Marolf, Thiemann.

The years immediately following the discovery of the loop formalism were mostly dedicated to understanding the loop representation by studying it in simpler contexts, such as $2+1$ general relativity [114, [45, [2]], Maxwell [22], linearized gravity [23], and, much later, 2d Yang-Mills theory [20].

\section{Classical limit: weaves}

Ashtekar, Rovelli, Smolin.

The first indication that the theory predicts that Planck scale discreteness came from studying the states that approximate geometries flat on large scale [24]. These states, denoted "weaves", have a "polymer" like structure at short scale, and can be viewed as a formalization of Wheeler's "spacetime foam".

\section{$1992 C^{*}$ algebraic framework}

Ashtekar, Isham.

In [13], Abhay Ashtekar and Chris Isham showed that the loop transform introduced in gravity by Rovelli and Smolin could be given a rigorous mathematical foundation, and set the basis for a mathematical systematization of the loop ideas, based on $C^{*}$ algebra ideas.

1993 Gravitons as embroideries over the weave Iwasaki, Rovelli.

In [124] Junichi Iwasaki and Rovelli studied the representation of gravitons

Living Reviews in Relativity (1998-1) http://www.livingreviews.org 
in loop quantum gravity. These appear as topological modifications of the fabric of the spacetime weave.

1993 Alternative versions

Di Bartolo, Gambini, Griego, Pullin.

Some versions of the loop quantum gravity alternative to the "orthodox" version have been developed. In particular, the authors above have developed the so called "extended" loop representation. See [ [80, [78].

1994 Fermions,

Morales-Tecotl, Rovelli.

Matter coupling was beginning to be explored in [149, 150]. Later, matter's kinematics was studied by Baez and Krasnov [13], 36], while Thiemann extended his results on the dynamics to the coupled Einstein Yang-Mills system in [201] .

1994 The $d \mu_{0}$ measure and the scalar product Ashtekar, Lewandowski, Baez.

In [15, 16, 177] Ashtekar and Lewandowski set the basis of the differential formulation of loop quantum gravity by constructing its two key ingredients: a diffeomorphism invariant measure on the space of (generalized) connections, and the projective family of Hilbert spaces associated to graphs. Using these techniques, they were able to give a mathematically rigorous construction of the state space of the theory, solving long standing problems deriving from the lack of a basis (the insufficient control on the algebraic identities between loop states). Using this, they defined a consistent scalar product and proved that the quantum operators in the theory were consistent with all identities. John Baez showed how the measure can be used in the context of conventional connections, extended it to the non-gauge invariant states (allowing the $E$ operator to be defined) and developed the use of the graph techniques [29, 30, [28]. Important contributions to the understanding of the measure were also given by Marolf and Mourão [148].

\section{Discreteness of area and volume eigenvalues}

\section{Rovelli, Smolin.}

In my opinion, the most significant result of loop quantum gravity is the discovery that certain geometrical quantities, in particular area and volume, are represented by operators that have discrete eigenvalues. This was found by Rovelli and Smolin in [186], where the first set of these eigenvalues was computed. Shortly after, this result was confirmed and extended by a number of authors, using very diverse techniques. In particular, Renate Loll [141, 142] used lattice techniques to analyze the volume operator and corrected a numerical error in [186]. Ashtekar and Lewandowski [137, 18] recovered and completed the computation of the spectrum of the area using the connection representation, and new regularization techniques. Frittelli, Lehner and Rovelli [84] recovered the

Living Reviews in Relativity (1998-1)

http://www.livingreviews.org 
Ashtekar-Lewandowski terms of the spectrum of the area, using the loop representation. DePietri and Rovelli [77] computed general eigenvalues of the volume. Complete understanding of the precise relation between different versions of the volume operator came from the work of Lewandowski [138].

1995 Spin networks - solution of the overcompleteness problem Rovelli, Smolin, Baez.

A long standing problem with the loop basis was its overcompleteness. A technical, but crucial step in understanding the theory has been the discovery of the spin-network basis, which solves this overcompleteness. This step was taken by Rovelli and Smolin in [187] and was motivated by the work of Roger Penrose [152, 151], by analogous bases used in lattice gauge theory and by ideas of Lewandowski [136]. Shortly after, the spin network formalism was cleaned up and clarified by John Baez [34, 3.5]. After the introduction of the spin network basis, all problems deriving from the incompleteness of the loop basis are trivially solved, and the scalar product could be defined also algebraically [77].

1995 Lattice

Loll, Reisenberger, Gambini, Pullin.

Various lattice versions of the theory have appeared in [140, 15.9, 94, 7.9].

1995 Algebraic formalism / Differential formalism

DePietri, Rovelli / Ashtekar, Lewandowski, Marolf, Mourão, Thiemann.

The cleaning and definitive setting of the two main versions of the formalisms was completed in [ [77] for the algebraic formalism (the direct descendent of the old loop representation); and in [IT.] for the differential formalism (based on the Ashtekar-Isham $C^{*}$ algebraic construction, on the Ashtekar-Lewandowski measure, on Don Marolf's work on the use of formal group integration for solving the constraints [144, 146, 147], and on several mathematical ideas by José Mourão).

1996 Equivalence of the algebraic and differential formalisms DePietri.

In [76], Roberto DePietri proved the equivalence of the two formalisms, using ideas from Thiemann [207] and Lewandowski [138] .

\section{Hamiltonian constraint}

Thiemann.

The first version of the loop hamiltonian constraint is in [183, 184]. The definition of the constraint has then been studied and modified repeatedly, in a long sequence of works, by Brügmann, Pullin, Blencowe, Borissov and others [117, 48, 60, 58, 57, 5.9, 157, .92, 4.9]. An important step was made by Rovelli and Smolin in [18.5] with the realization that certain regularized loop operators have finite limits on knot states (see [1339]). The search culminated with the work of Thomas Thiemann, who

Living Reviews in Relativity (1998-1)

http://www.livingreviews.org 
was able to construct a rather well-defined hamiltonian operator whose constraint algebra closes [208, 2012, 2013]. Variants of this constraint have been suggested in [1.92, 160] and elsewhere.

1996 Real theory: solution of the reality conditions problem Barbero, Thiemann.

As often stressed by Karel Kuchař, implementing the complicated reality condition of the complex connection into the quantum theory was, until 1996, the main open problem in the loop approach. ${ }^{3}$ Following the directions advocated by Fernando Barbero [40, 41, 42, 43], namely to use the real connection in the Lorentzian theory, Thiemann found an elegant way to completely bypass the problem.

\section{Black hole entropy}

Krasnov, Rovelli.

A derivation of the Bekenstein-Hawking formula for the entropy of a black hole from loop quantum gravity was obtained in [1]6], on the basis of the ideas of Kirill Krasnov [133, 1134]. Recently, Ashtekar, Baez, Corichi and Krasnov have announced an alternative derivation [112].

\section{Anomalies}

\section{Lewandowski, Marolf, Pullin, Gambini.}

These authors have recently completed an extensive analysis of the issue of the closure of the quantum constraint algebra and its departures from the corresponding classical Poisson algebra [13.9, 90], following earlier pioneering work in this direction by Brügmann, Pullin, Borissov and others [55, 61, 88, 51]. This analysis has raised worries that the classical limit of Thiemann's hamiltonian operator might fail to yield classical general relativity, but the matter is still controversial.

\section{Sum over surfaces}

Reisenberger Rovelli.

A "sum over histories" spacetime formulation of loop quantum gravity was derived in [18], 160] from the canonical theory. The resulting covariant theory turns out to be a sum over topologically inequivalent surfaces, realizing earlier suggestions by $\boldsymbol{B a e z}$ [3], 28, 34, 25], Reisenberger [15.9, [158] and Iwasaki [12:3] that a covariant version of loop gravity should look like a theory of surfaces. Baez has studied the general structure of theories defined in this manner [26]. Smolin and Markoupolou have explored the extension of the construction to the Lorentzian case, and the possibility of altering the spin network evolution rules [14:3].

\footnotetext{
3 "The loop people have a credit card called reality conditions, and whenever they solve a problem, they charge the card, but one day the bill comes and the whole thing breaks down like a card house" [1.35].
}

Living Reviews in Relativity (1998-1)

http: //www . livingreviews .org 


\section{Resources}

- A valuable resource for finding relevant literature is the comprehensive "Bibliography of Publications Related to Classical and Quantum Gravity in terms of Connection and Loop Variables", organized chronologically. The original version was compiled by Peter Hübner in 1989. It has subsequently been updated by Gabriella Gonzales, Bernd Brügmann, Monica Pierri and Troy Shiling. Presently, it is being kept updated by Christopher Beetle and Alejandro Corichi. The latest version can be found on the net in [45].

- This "living review" may serve as an up to date introduction to quantum gravity in the loop formalism. More detailed (but less up to date) presentations are listed below.

- Ashtekar's book [10] may serve as a valuable basic introductory course on Ashtekar variables, particularly for relativists and mathematicians. The part of the book on the loop representation is essentially an authorized reprint of parts of the original Rovelli Smolin article [184]. For this quantum part, I recommend looking at the article, rather than the book, because the article is more complete.

- A simpler and more straightforward introduction to the Ashtekar variables and basic loop ideas can be found in the Rovelli's review paper [164]. This is more oriented to a reader with a physics background.

- A recent general introduction to the new variables which includes several of the recent mathematical developments in the quantum theory is given by Ashtekar's Les Houches 1992 lectures [II].

- A particularly interesting collection of papers can be found in the volume [32] edited by John Baez. The other book by Baez, and Muniain [37], is a simple and pleasant introduction to several ideas and techniques in the field.

- The last and up to date book on the loop representation is the book by Gambini and Pullin [9.3], especially good in lattice techniques and in the variant of loop quantum gravity called the "extended loop representation" [ [80, [8] (which is nowadays a bit out of fashion, but remains an intriguing alternative to "orthodox" loop quantum gravity).

- The two standard references for a complete presentation of the basics of the theory are: DePietri and Rovelli [77] for the algebraic formulation; and Ashtekar, Lewandowski, Marolf, Mourao and Thiemann $\left(A L M^{2} T\right)$ [1.9] for the differential formulation.

- Besides the many conferences on gravity, the loop gravity community has met twice in Warsaw, in the "Workshop on Canonical and Quantum Gravity". Hopefully, this will become a recurrent meeting. This may be the

Living Reviews in Relativity (1998-1) http://www.livingreviews.org 
right place to go for learning what is going on in the field. For an informal account of the last of these meetings (August 1997), see [16T].

- Some of the main institutions where loop quantum gravity is studied are

- The "Center for Gravity and Geometry" at Penn State University, USA. I recommend their invaluable web page [156], maintained by Jorge Pullin, for finding anything you need from the web.

- Pittsburgh University, USA [15.5].

- University of California at Riverside, USA. John Baez moderates an interesting newsgroup, sci.physics.research, with news from the field. See [27].

- Albert Einstein Institute, Potsdam, Berlin, Europe [2].

- Warsaw University, Warsaw, Europe.

- Imperial College, London, Europe [ए6].

- Syracuse University, USA [1.99].

- Montevideo University, Uruguay. 


\section{Main Ideas and Physical Inputs}

The main physical hypotheses on which loop quantum gravity relies are only general relativity and quantum mechanics. In other words, loop quantum gravity is a rather conservative "quantization" of general relativity, with its traditional matter couplings. In this sense, it is very different from string theory, which is based on a strong physical hypothesis with no direct experimental support (the world is made by strings).

Of course "quantization" is far from a univocal algorithm, particularly for a nonlinear field theory. Rather, it is a poorly understood inverse problem (find a quantum theory with the given classical limit). More or less subtle choices are made in constructing the quantum theory. I discuss these choices below.

\subsection{Quantum field theory on a differentiable manifold}

The main idea beyond loop quantum gravity is to take general relativity seriously. We have learned with general relativity that the spacetime metric and the gravitational field are the same physical entity. Thus, a quantum theory of the gravitational field is a quantum theory of the spacetime metric as well. It follows that quantum gravity cannot be formulated as a quantum field theory over a metric manifold, because there is no (classical) metric manifold whatsoever in a regime in which gravity (and therefore the metric) is a quantum variable.

One could conventionally split the spacetime metric into two terms: one to be considered a background, which gives a metric structure to spacetime; the other to be treated as a fluctuating quantum field. This, indeed, is the procedure on which old perturbative quantum gravity, perturbative strings, as well as current non-perturbative string theories (M-theory), are based. In following this path, one assumes, for instance, that the causal structure of spacetime is determined by the underlying background metric alone, and not by the full metric. Contrary to this, in loop quantum gravity we assume that the identification between the gravitational field and the metric-causal structure of spacetime holds, and must be taken into account, in the quantum regime as well. Thus, no split of the metric is made, and there is no background metric on spacetime.

We can still describe spacetime as a (differentiable) manifold (a space without metric structure), over which quantum fields are defined. A classical metric structure will then be defined by expectation values of the gravitational field operator. Thus, the problem of quantum gravity is the problem of understanding what is a quantum field theory on a manifold, as opposed to quantum field theory on a metric space. This is what gives quantum gravity its distinctive flavor, so different from ordinary quantum field theory. In all versions of ordinary quantum field theory, the metric of spacetime plays an essential role in the construction of the basic theoretical tools (creation and annihilation operators, canonical commutation relations, gaussian measures, propagators ...); these tools cannot be used in quantum field over a manifold.

Living Reviews in Relativity (1998-1) http://www.livingreviews.org 
Technically, the difficulty due to the absence of a background metric is circumvented in loop quantum gravity by defining the quantum theory as a representation of a Poisson algebra of classical observables which can be defined without using a background metric. The idea that the quantum algebra at the basis of quantum gravity is not the canonical commutation relation algebra, but the Poisson algebra of a different set of observables, has long been advocated by Chris Isham [117], whose ideas have been very influential in the birth of loop quantum gravity.t The algebra on which loop gravity is the loop algebra [184]. The particular choice of this algebra is not harmless, as I discuss below.

\subsection{One additional assumption}

In choosing the loop algebra as the basis for the quantization, we are essentially assuming that Wilson loop operators are well defined in the Hilbert space of the theory; in other words, that certain states concentrated on one dimensional structures (loops and graphs) have finite norm. This is a subtle non trivial assumption entering the theory. It is the key assumption that characterizes loop gravity. If the approach turned out to be wrong, it will likely be because this assumption is wrong. The Hilbert space resulting from adopting this assumption is not a Fock space. Physically, the assumption corresponds to the idea that quantum states can be decomposed on a basis of "Faraday lines" excitations (as Minkowski QFT states can be decomposed on a particle basis).

Furthermore, this is an assumption that fails in conventional quantum field theory, because in that context well defined operators and finite norm states need to be smeared in at least three dimensions, and one-dimensional objects are too singular. 0 The fact that at the basis of loop gravity there is a mathematical assumption that fails for conventional Yang-Mills quantum field theory is probably at the origin of some of the resistance that loop quantum gravity encounters among some high energy theorists. What distinguishes gravity from Yang-Mills theories, however, and makes this assumption viable in gravity, even if it fails for Yang-Mills theory, is diffeomorphism invariance. The loop states are singular states that span a "huge" non-separable state space. (Nonperturbative) diffeomorphism invariance plays two roles. First, it wipes away the infinite redundancy. Second, it "smears" a loop state into a knot state, so that the physical states are not really concentrated in one dimension, but are, in a sense, smeared all over the entire manifold by the nonperturbative diffeomorphisms. This will be more clear in the next section.

\footnotetext{
${ }^{4}$ Loop Quantum Gravity is an attempt to solve the last problem in Isham's lectures [17].

${ }^{5}$ The assumption does not fail, however, in two-dimensional Yang-Mills theory, which is invariant under area preserving diffeomorphisms, and where loop quantization techniques were successfully employed [ZiI].
}

Living Reviews in Relativity (1998-1)

http://www.livingreviews.org 


\subsection{Physical meaning of diffeomorphism invariance, and its implementation in the quantum theory}

Conventional field theories are not invariant under a diffeomorphism acting on the dynamical fields. (Every field theory, suitably formulated, is trivially invariant under a diffeomorphism acting on everything.) General relativity, on the contrary, is invariant under such transformations. More precisely, every general relativistic theory has this property. Thus, diffeomorphism invariance is not a feature of just the gravitational field: It is a feature of physics, once the existence of relativistic gravity is taken into account. Thus, one can say that the gravitational field is not particularly "special" in this regard, but that diff-invariance is a property of the physical world that can be disregarded only in the approximation in which the dynamics of gravity is neglected. What is this property? What is the physical meaning of diffeomorphism invariance?

Diffeomorphism invariance is the technical implementation of a physical idea, due to Einstein. The idea is a deep modification of the pre-general-relativistic (pre-GR) notions of space and time. In pre-GR physics, we assume that physical objects can be localized in space and time with respect to a fixed non-dynamical background structure. Operationally, this background spacetime can be defined by means of physical reference-system objects, but these objects are considered as dynamically decoupled from the physical system that one studies. This conceptual structure fails in a relativistic gravitational regime. In general relativistic physics, the physical objects are localized in space and time only with respect to each other. Therefore if we "displace" all dynamical objects in spacetime at once, we are not generating a different state, but an equivalent mathematical description of the same physical state. Hence, diffeomorphism invariance.

Accordingly, a physical state in GR is not "located" somewhere [180], 169, [77] (unless an appropriate gauge fixing is made). Pictorially, GR is not physics over a stage, it is the dynamical theory of (or including) the stage itself.

Loop quantum gravity is an attempt to implement this subtle relational notion of spacetime localization in quantum field theory. In particular, the basic quantum field theoretical excitations cannot be localized somewhere (localized with respect to what?) as, say, photons are. They are quantum excitations of the "stage" itself, not excitations over a stage. Intuitively, one can understand from this discussion how knot theory plays a role in the theory. First, we define quantum states that correspond to loop-like excitations of the gravitational field, but then, when factoring away diffeomorphism invariance, the location of the loop becomes irrelevant. The only remaining information contained in the loop is then its knotting (a knot is a loop up to its location). Thus, diffeomorphism invariant physical states are labeled by knots. A knot represents an elementary quantum excitation of space. It is not here or there, since it is the space with respect to which here and there can be defined. A knot state is an elementary quantum of space.

In this manner, loop quantum gravity ties the new notion of space and time introduced by general relativity with quantum mechanics. As I will illustrate later on, the existence of such elementary quanta of space is then made concrete

Living Reviews in Relativity (1998-1) http://www.livingreviews.org 
by the quantization of the spectra of geometrical quantities.

\subsection{Problems not addressed}

Quantum gravity is an open problem that has been investigated for over seventy years now. When one contemplates two deep problems, one is tempted to believe that they are related. In the history of physics, there are surprising examples of two deep problems solved by one stroke (the unification of electricity and magnetism and the nature of light, for instance); but also many examples in which a great hope to solve more than one problem at once was disappointed (finding the theory of strong interactions and getting rid of quantum field theory infinities, for instance). Quantum gravity has been asked, at one time or another, to address almost every deep open problem in theoretical physics (and beyond). Here is a list of problems that have been connected to quantum gravity in the past, but about which loop quantum gravity has little to say:

Interpretation of quantum mechanics. Loop quantum gravity is a standard quantum (field) theory. Pick your favorite interpretation of quantum mechanics, and use it for interpreting the quantum aspects of the theory. I will refer to two such interpretations below: When discussing the quantization of area and volume, I will use the relation between eigenvalues and outcomes of measurements performed with classical physical apparatusses; when discussing evolution, I will refer to the histories interpretation. The peculiar way of describing time evolution in a general relativistic theory may require some appropriate variants of standard interpretations, such as generalized canonical quantum theory [166, 168, 165] or Hartle's generalized quantum mechanics [102]. But loop quantum gravity has no help to offer the scientists who have speculated that quantum gravity will solve the measurement problem. On the other hand, the spacetime formulation of loop quantum gravity that has recently been developed (see Section 6.10) is naturally interpreted in terms of histories interpretations [102, ए18, ए19, ए22, ए21]. Furthermore, I think that solving the problem of the interpretation of quantum mechanics might require relational ideas connected with the relational nature of spacetime revealed by general relativity [1779, 1180]].

Quantum cosmology. There is widespread confusion between quantum cosmology and quantum gravity. Quantum cosmology is the theory of the entire universe as a quantum system without external observer [101]: With or without gravity, makes no difference. Quantum gravity is the theory of one dynamical entity: the quantum gravitational field (or the spacetime metric), just one field among the many. Precisely as for the theory of the quantum electromagnetic field, we can always assume that we have a classical observer with classical measuring apparatusses measuring gravitational phenomena, and therefore study quantum gravity disregarding quantum cosmology. For instance, the physics of a Planck size small cube

Living Reviews in Relativity (1998-1)

http://www.livingreviews.org 
is governed by quantum gravity and, presumably, has no cosmological implications.

Unifications of all interactions or "Theory of Everything". A common criticism of loop quantum gravity is that it does not unify all interactions. But the idea that quantum gravity can be understood only in conjunction with other fields is an interesting hypothesis, not an established truth.

Mass of the elementary particles. As far as I see, nothing in loop quantum gravity suggests that one could compute masses from quantum gravity.

Origin of the Universe. It is likely that a sound quantum theory of gravity will be needed to understand the physics of the Big Bang. The converse is probably not true: We should be able to understand the small scale structure of spacetime even if we do not understand the origin of the Universe.

Arrow of time. Roger Penrose has argued for some time that it should be possible to trace the time asymmetry in the observable Universe to quantum gravity.

Physics of the mind. Penrose has also speculated that quantum gravity is responsible for the wave function collapse, and, indirectly, governs the physics of the mind [154].

A problem that has been repeatedly tied to quantum gravity, and which loop quantum gravity might be able to address, is the problem of the ultraviolet infinities in quantum field theory. The very peculiar nonperturbative short scale structure of loop quantum gravity introduces a physical cutoff. Since physical spacetime itself comes in quanta in the theory, there is literally no space in the theory for the very high momentum integrations that originate from the ultraviolet divergences. Lacking a complete and detailed calculation scheme, however, one cannot yet claim with confidence that the divergences, chased from the door, will not reenter from the window. 


\section{The Formalism}

Here, I begin the technical description of the basics of loop quantum gravity. The starting point of the construction of the quantum theory is classical general relativity, formulated in terms of the Sen-Ashtekar-Barbero connection [IT0, 8, 4II]. Detailed introductions to the (complex) Ashtekar formalism can be found in the book [10], in the review article [164], and in the conference proceedings [81]. The real version of the theory is presently the most widely used.

Classical general relativity can be formulated in phase space form as follows [10, 41]. We fix a three-dimensional manifold $M$ (compact and without boundaries) and consider a smooth real $S U(2)$ connection $A_{a}^{i}(x)$ and a vector density $\tilde{E}_{i}^{a}(x)$ (transforming in the vector representation of $\left.S U(2)\right)$ on $M$. We use $a, b, \ldots=1,2,3$ for spatial indices and $i, j, \ldots=1,2,3$ for internal indices. The internal indices can be viewed as labeling a basis in the Lie algebra of $S U(2)$ or in the three axis of a local triad. We indicate coordinates on $M$ with $x$. The relation between these fields and conventional metric gravitational variables is as follows: $\tilde{E}_{i}^{a}(x)$ is the (densitized) inverse triad, related to the three-dimensional metric $g_{a b}(x)$ of constant-time surfaces by

$$
g g^{a b}=\tilde{E}_{i}^{a} \tilde{E}_{i}^{b},
$$

where $g$ is the determinant of $g_{a b}$; and

$$
A_{a}^{i}(x)=\Gamma_{a}^{i}(x)+\gamma k_{a}^{i}(x)
$$

$\Gamma_{a}^{i}(x)$ is the spin connection associated to the triad, (defined by $\partial_{[a} e_{b]}^{i}=\Gamma_{[a}^{i} e_{b] j}$, where $e_{a}^{i}$ is the triad). $k_{a}^{i}(x)$ is the extrinsic curvature of the constant time three surface.

In (2), $\gamma$ is a constant, denoted the Immirzi parameter, that can be chosen arbitrarily (It will enter the hamiltonian constraint.) [133, Ш4, Ш15]. Different choices for $\gamma$ yield different versions of the formalism, all equivalent in the classical domain. If we choose $\gamma$ to be equal to the imaginary unit, $\gamma=\sqrt{-1}$, then $A$ is the standard Ashtekar connection, which can be shown to be the projection of the selfdual part of the four-dimensional spin connection on the constant time surface. If we choose $\gamma=1$, we obtain the real Barbero connection. The hamiltonian constraint of Lorentzian general relativity has a particularly simple form in the $\gamma=\sqrt{-1}$ formalism; while the hamiltonian constraint of Euclidean general relativity has a simple form when expressed in terms of the $\gamma=1$ real connection. Other choices of $\gamma$ are viable as well. In particular, it has been argued that the quantum theory based on different choices of $\gamma$ are genuinely physical inequivalent, because they yield "geometrical quanta" of different magnitude [18.9]. Apparently, there is a unique choice of $\gamma$ yielding the correct $1 / 4$

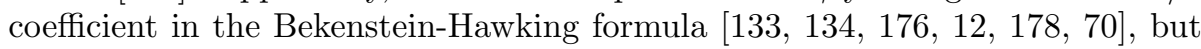
the matter is still under discussion.

The spinorial version of the Ashtekar variables is given in terms of the Pauli matrices $\sigma_{i}, i=1,2,3$, or the $s u(2)$ generators $\tau_{i}=-\frac{\mathrm{i}}{2} \sigma_{i}$, by

$$
\tilde{E}^{a}(x)=-\mathrm{i} \tilde{E}_{i}^{a}(x) \sigma_{i}=2 \tilde{E}_{i}^{a}(x) \tau_{i},
$$

Living Reviews in Relativity (1998-1)

http://www.livingreviews.org 


$$
A_{a}(x)=-\frac{\mathrm{i}}{2} A_{a}^{i}(x) \sigma_{i}=A_{a}^{i}(x) \tau_{i}
$$

Thus, $A_{a}(x)$ and $\tilde{E}^{a}(x)$ are $2 \times 2$ anti-hermitian complex matrices.

The theory is invariant under local $S U(2)$ gauge transformations, threedimensional diffeomorphisms of the manifold on which the fields are defined, as well as under (coordinate) time translations generated by the hamiltonian constraint. The full dynamical content of general relativity is captured by the three constraints that generate these gauge invariances [190, 10].

As already mentioned, the Lorentzian hamiltonian constraint does not have a simple polynomial form if we use the real connection (2). For a while, this fact was considered an obstacle to defining the quantum hamiltonian constraint; therefore the complex version of the connection was mostly used. However, Thiemann has recently succeeded in constructing a Lorentzian quantum hamiltonian constraint [208, 202, 203] in spite of the non-polynomiality of the classical expression. This is the reason why the real connection is now widely used. This choice has the advantage of eliminating the old "reality conditions" problem, namely the problem of implementing non-trivial reality conditions in the quantum theory.

\subsection{Loop algebra}

Certain classical quantities play a very important role in the quantum theory. These are: the trace of the holonomy of the connection, which is labeled by loops on the three manifold; and the higher order loop variables, obtained by inserting the $E$ field (in $n$ distinct points, or "hands") into the holonomy trace. More precisely, given a loop $\alpha$ in $M$ and the points $s_{1}, s_{2}, \ldots, s_{n} \in \alpha$ we define:

$$
\begin{aligned}
\mathcal{T}[\alpha] & =-\operatorname{Tr}\left[U_{\alpha}\right], \\
\mathcal{T}^{a}[\alpha](s) & =-\operatorname{Tr}\left[U_{\alpha}(s, s) \tilde{E}^{a}(s)\right]
\end{aligned}
$$

and, in general

$$
\begin{aligned}
& \mathcal{T}^{a_{1} a_{2}}[\alpha]\left(s_{1}, s_{2}\right)= \\
& \quad=-\operatorname{Tr}\left[U_{\alpha}\left(s_{1}, s_{2}\right) \tilde{E}^{a_{2}}\left(s_{2}\right) U_{\alpha}\left(s_{2}, s_{1}\right) \tilde{E}^{a_{1}}\left(s_{1}\right)\right] \\
& \mathcal{T}^{a_{1} \ldots a_{N}}[\alpha]\left(s_{1} \ldots s_{N}\right)= \\
& \quad=-\operatorname{Tr}\left[U_{\alpha}\left(s_{1}, s_{N}\right) \tilde{E}^{a_{N}}\left(s_{N}\right) U_{\alpha}\left(s_{N}, s_{N-1}\right) \ldots \tilde{E}^{a_{1}}\left(s_{1}\right)\right]
\end{aligned}
$$

where $U_{\alpha}\left(s_{1}, s_{2}\right) \sim \mathcal{P} \exp \left\{\int_{s_{1}}^{s_{2}} A_{a}(\alpha(s)) d s\right\}$ is the parallel propagator of $A_{a}$ along $\alpha$, defined by

$$
\frac{d}{d s} U_{\alpha}(1, s)=\frac{d \alpha_{a}(s)}{d s} A_{a}(\alpha(s)) U_{\alpha}(1, s)
$$

(See [r7] for more details.) These are the loop observables, introduced in Yang Mills theories in [95, 96], and in gravity in [183, 184].

The loop observables coordinatize the phase space and have a closed Poisson algebra, denoted by the loop algebra. This algebra has a remarkable geometrical

Living Reviews in Relativity (1998-1) http://www.livingreviews.org 
flavor. For instance, the Poisson bracket between $\mathcal{T}[\alpha]$ and $\mathcal{T}^{a}[\beta](s)$ is non vanishing only if $\beta(s)$ lies over $\alpha$; if it does, the result is proportional to the holonomy of the Wilson loops obtained by joining $\alpha$ and $\beta$ at their intersection (by rerouting the 4 legs at the intersection). More precisely

$$
\left\{\mathcal{T}[\alpha], \mathcal{T}^{a}[\beta](s)\right\}=\Delta^{a}[\alpha, \beta(s)]\left[\mathcal{T}[\alpha \# \beta]-\mathcal{T}\left[\alpha \# \beta^{-1}\right]\right] .
$$

Here

$$
\Delta^{a}[\alpha, x]=\int d s \frac{d \alpha^{a}(s)}{d s} \delta^{3}(\alpha(s), x)
$$

is a vector distribution with support on $\alpha$ and $\alpha \# \beta$ is the loop obtained starting at the intersection between $\alpha$ and $\beta$, and following first $\alpha$ and then $\beta$. $\beta^{-1}$ is $\beta$ with reversed orientation.

A (non-SU(2) gauge invariant) quantity that plays a role in certain aspects of the theory, particularly in the regularization of certain operators, is obtained by integrating the $E$ field over a two dimensional surface $S$

$$
E[S, f]=\int_{S} d S_{a} \tilde{E}_{i}^{a} f^{i},
$$

where $f$ is a function on the surface $S$, taking values in the Lie algebra of $S U(2)$. As an alternative to the full loop observables (5,66,7), one can also take the holonomies and $E[S, f]$ as elementary variables [[16, [8]; this is more natural to do, for instance, in the $\mathrm{C}^{*}$-algebric approach [1]3].

\subsection{Loop quantum gravity}

The kinematics of a quantum theory is defined by an algebra of "elementary" operators (such as $x$ and $i \hbar d / d x$, or creation and annihilation operators) on a Hilbert space $\mathcal{H}$. The physical interpretation of the theory is based on the connection between these operators and classical variables, and on the interpretation of $\mathcal{H}$ as the space of the quantum states. The dynamics is governed by a hamiltonian, or, as in general relativity, by a set of quantum constraints, constructed in terms of the elementary operators. To assure that the quantum Heisenberg equations have the correct classical limit, the algebra of the elementary operator has to be isomorphic to the Poisson algebra of the elementary observables. This yields the heuristic quantization rule: "promote Poisson brackets to commutators". In other words, define the quantum theory as a linear representation of the Poisson algebra formed by the elementary observables. For the reasons illustrated in section [5, the algebra of elementary observables we choose for the quantization is the loop algebra, defined in section 6.1. Thus, the kinematic of the quantum theory is defined by a unitary representation of the loop algebra. Here, I construct such representation following a simple path.

We can start "à la Schrödinger" by expressing quantum states by means of the amplitude of the connection, namely by means of functionals $\Psi(A)$ of the (smooth) connection. These functionals form a linear space, which we promote to a Hilbert space by defining a inner product. To define the inner product, we

Living Reviews in Relativity (1998-1)

http://www.livingreviews.org 
choose a particular set of states, which we denote "cylindrical states" and begin by defining the scalar product between these.

Pick a graph $\Gamma$, say with $n$ links, denoted $\gamma_{1} \ldots \gamma_{n}$, immersed in the manifold $M$. For technical reasons, we require the links to be analytic. Let $U_{i}(A)=$ $U_{\gamma_{i}}, i=1, \ldots, n$ be the parallel transport operator of the connection $A$ along $\gamma_{i}$. $U_{i}(A)$ is an element of $S U(2)$. Pick a function $f\left(g_{1} \ldots g_{n}\right)$ on $[S U(2)]^{n}$. The graph $\Gamma$ and the function $f$ determine a functional of the connection as follows

$$
\psi_{\Gamma, f}(A)=f\left(U_{1}(A), \ldots, U_{n}(A)\right) .
$$

(These states are called cylindrical states because they were introduced in [15., [16, [7] as cylindrical functions for the definition of a cylindrical measure.) Notice that we can always "enlarge the graph", in the sense that if $\Gamma$ is a subgraph of $\Gamma^{\prime}$, we can always write

$$
\psi_{\Gamma, f}(A)=\psi_{\Gamma^{\prime}, f^{\prime}}(A) .
$$

by simply choosing $f^{\prime}$ independent from the $U_{i}$ 's of the links which are in $\Gamma^{\prime}$ but not in $\Gamma$. Thus, given any two cylindrical functions, we can always view them as having the same graph (formed by the union of the two graphs). Given this observation, we define the scalar product between any two cylindrical functions [1.36, ㅍ.5, 16, 17] by

$$
\left(\psi_{\Gamma, f}, \psi_{\Gamma, h}\right)=\int_{S U(2)^{n}} d g_{1} \ldots d g_{n} \overline{f\left(g_{1} \ldots g_{n}\right)} h\left(g_{1} \ldots g_{n}\right) .
$$

where $d g$ is the Haar measure on $S U(2)$. This scalar product extends by linearity to finite linear combinations of cylindrical functions. It is not difficult to show that (14) defines a well defined scalar product on the space of these linear combinations. Completing the space of these linear combinations in the Hilbert norm, we obtain a Hilbert space $\mathcal{H}$. This is the (unconstrained) quantum state space of loop gravity. $\mathcal{H}$ carries a natural unitary representation of the diffeomorphism group and of the group of the local $S U(2)$ transformations, obtained transforming the argument of the functionals. An important property of the scalar product (14) is that it is invariant under both these transformations.

$\mathcal{H}$ is non-separable. At first sight, this may seem a serious obstacle to its physical interpretation. But we will see below that, after factoring away diffeomorphism invariance, we may obtain a separable Hilbert space (see section 6.8). Also, standard spectral theory holds on $\mathcal{H}$, and it turns out that using spin networks (discussed below) one can express $\mathcal{H}$ as a direct sum over finite dimensional subspaces which have the structure of Hilbert spaces of spin systems; this makes practical calculations very manageable.

\footnotetext{
${ }^{6}$ This construction of $\mathcal{H}$ as the closure of the space of the cylindrical functions of smooth connections in the scalar product (14) shows that $\mathcal{H}$ can be defined without the need of recurring to $C^{*}$ algebraic techniques, distributional connections or the Ashtekar-Lewandowski measure. The casual reader, however, should be warned that the resulting $\mathcal{H}$ topology is different than the natural topology on the space of connections: if a sequence $\Gamma_{n}$ of graphs converges pointwise to a graph $\Gamma$, the corresponding cylindrical functions $\psi_{\Gamma_{n}, f}$ do not converge to $\psi_{\Gamma, f}$ in the $\mathcal{H}$ Hilbert space topology.
}

Living Reviews in Relativity (1998-1) http://www.livingreviews.org 
Finally, we will use a Dirac notation and write

$$
\Psi(A)=\langle A \mid \Psi\rangle,
$$

in the same manner in which one may write $\psi(x)=\langle x \mid \Psi\rangle$ in ordinary quantum mechanics. As in that case, however, we should remember that $|A\rangle$ is not a normalizable state.

\subsection{Loop states and spin network states}

A subspace $\mathcal{H}_{0}$ of $\mathcal{H}$ is formed by states invariant under $S U(2)$ gauge transformations. We now define an orthonormal basis in $\mathcal{H}_{0}$. This basis represents a very important tool for using the theory. It was introduced in [187] and developed in [34, 35]; it is denoted spin network basis.

First, given a loop $\alpha$ in $M$, there is a normalized state $\psi_{\alpha}(A)$ in $\mathcal{H}$, which is obtained by taking $\Gamma=\alpha$ and $f(g)=-\operatorname{Tr}(g)$. Namely

$$
\psi_{\alpha}(A)=-\operatorname{Tr} U_{\alpha}(A) .
$$

We introduce a Dirac notation for the abstract states, and denote this state as $|\alpha\rangle$. These sates are called loop states. Using Dirac notation, we can write

$$
\psi_{\alpha}(A)=\langle A \mid \alpha\rangle .
$$

It is easy to show that loop states are normalizable. Products of loop states are normalizable as well. Following tradition, we denote with $\alpha$ also a multiloop, namely a collection of (possibly overlapping) loops $\left\{\alpha_{1}, \ldots, \alpha_{n},\right\}$, and we call

$$
\psi_{\alpha}(A)=\psi_{\alpha_{1}}(A) \times \ldots \times \psi_{\alpha_{n}}(A)
$$

a multiloop state. (Multi-)loop states represented the main tool for loop quantum gravity before the discovery of the spin network basis. Linear combinations of multiloop states (over-) span $\mathcal{H}$, and therefore a generic state $\psi(A)$ is fully characterized by its projections on the multiloop states, namely by

$$
\psi(\alpha)=\left(\psi_{\alpha}, \psi\right) .
$$

The "old" loop representation was based on representing quantum states in this manner, namely by means of the functionals $\psi(\alpha)$ over loop space defined in(19). Equation (19) can be explicitly written as an integral transform, as we will see in section 6.7.

Next, consider a graph $\Gamma$. A "coloring" of $\Gamma$ is given by the following.

1. Associate an irreducible representation of $S U(2)$ to each link of $\Gamma$. Equivalently, we may associate to each link $\gamma_{i}$ a half integer number $s_{i}$, the spin of the irreducible, or, equivalently, an integer number $p_{i}$, the "color" $p_{i}=2 s_{i}$.

Living Reviews in Relativity (1998-1) http://www.livingreviews.org 
2. Associate an invariant tensor $v$ in the tensor product of the representations $s_{1} \ldots s_{n}$, to each node of $\Gamma$ in which links with spins $s_{1} \ldots s_{n}$ meet. An invariant tensor is an object with $n$ indices in the representations $s_{1} \ldots s_{n}$ that transform covariantly. If $n=3$, there is only one invariant tensor (up to a multiplicative factor), given by the Clebsh-Gordon coefficient. An invariant tensor is also called an intertwining tensor. All invariant tensors are given by the standard Clebsch-Gordon theory. More precisely, for fixed $s_{1} \ldots s_{n}$, the invariant tensors form a finite dimensional linear space. Pick a basis $v_{j}$ is this space, and associate one of these basis elements to the node. Notice that invariant tensors exist only if the tensor product of the representations $s_{1} \ldots s_{n}$ contains the trivial representation. This yields a condition on the coloring of the links. For $n=3$, this is given by the well known Clebsh-Gordan condition: Each color is not larger than the sum of the other two, and the sum of the three colors is even.

We indicate a colored graph by $\{\Gamma, \vec{s}, \vec{v}\}$, or simply $S=\{\Gamma, \vec{s}, \vec{v}\}$, and denote it a "spin network". (It was Penrose who first had the intuition that this mathematics could be relevant for describing the quantum properties of the geometry, and who gave the first version of spin network theory [151, 152].)

Given a spin network $S$, we can construct a state $\Psi_{S}(A)$ as follows. We take the propagator of the connection along each link of the graph, in the representation associated to that link, and then, at each node, we contract the matrices of the representation with the invariant tensor. We obtain a state $\Psi_{S}(A)$, which we also write as

$$
\psi_{S}(A)=\langle A \mid S\rangle
$$

One can then show the following.

- The spin network states are normalizable. The normalization factor is computed in [77].

- They are $S U(2)$ gauge invariant.

- Each spin network state can be decomposed into a finite linear combination of products of loop states.

- The (normalized) spin network states form an orthonormal basis for the gauge $S U(2)$ invariant states in $\mathcal{H}$ (choosing the basis of invariant tensors appropriately).

- The scalar product between two spin network states can be easily computed graphically and algebraically. See [r7] for details.

The spin network states provide a very convenient basis for the quantum theory.

The spin network states defined above are $S U(2)$ gauge invariant. There exists also an extension of the spin network basis to the full Hilbert space (see for instance [18, 52], and references therein).

Living Reviews in Relativity (1998-1) http://www.livingreviews.org 


\subsection{Relation between spin network states and loop states and diagrammatic representation of the states}

A diagrammatic representation for the states in $\mathcal{H}$ is very useful in concrete calculations. First, associate to a loop state $|\alpha\rangle$ a diagram in $M$, formed by the loop $\alpha$ itself. Next, notice that we can multiply two loop states, obtaining a normalizable state. We represent the product of $n$ loop states by the diagram formed by the set of the $n$ (possibly overlapping) corresponding loops (we denote this set "multiloop"). Thus, linear combinations of multiloops diagrams represent states in $\mathcal{H}$. Representing states as linear combinations of multiloops diagrams makes computation in $\mathcal{H}$ easy.

Now, the spin network state defined by the graph with no nodes $\alpha$, with color 1 , is clearly, by definition, the loop state $|\alpha\rangle$, and we represent it by the diagram $\alpha$. The spin network state $|\alpha, n\rangle$ determined by the graph without nodes $\alpha$, with color $n$ can be obtained as follows. Draw $n$ parallel lines along the loop $\alpha$; cut all lines at an arbitrary point of $\alpha$, and consider the $n$ ! diagrams obtained by joining the legs after a permutation. The linear combination of these $n$ ! diagrams, taken with alternate signs (namely with the sign determined by the parity of the permutation) is precisely the state $|\alpha, n\rangle$. The reason for this key result can be found in the fact that an irreducible representation of $S U(2)$ can be obtained as the totally symmetric tensor product of the fundamental representation with itself. For details, see [77].

Next, consider a graph $\Gamma$ with nodes. Draw $n_{i}$ parallel lines along each link $\gamma_{i}$. Join pairwise the end points of these lines at each node (in an arbitrary manner), in such a way that each line is joined with a line from a different link (see Figure 11). In this manner, one obtains a multiloop diagram. Now antisymmetrize the $n_{i}$ parallel lines along each link, obtaining a linear combination of diagrams representing a state in $\mathcal{H}$. One can show that this state is a spin network state, where $n_{i}$ is the color of the links, and the color of the nodes is determined by the pairwise joining of the legs chosen [[r7]. Again, simple $S U(2)$ representation theory is behind this result.

More in detail, if a node is trivalent (has 3 adjacent links), then we can join legs pairwise only if the total number of the legs is even, and if the number of the legs in each link is smaller or equal than the sum of the number of the other two. This can be recognized immediately as the Clebsch-Gordan condition. If these conditions are satisfied, there is only a single way of joining legs. This corresponds to the fact that there is only one invariant tensor in the product of three irreducible of $S U(2)$. Higher valence nodes can be decomposed in trivalent "virtual" nodes, joined by "virtual" links. Orthogonal independent invariant tensors are obtained by varying over all allowed colorings of these virtual links (compatible with the Clebsch-Gordan conditions at the virtual nodes). Different decompositions of the node give different orthogonal bases. Thus the total (links and nodes) coloring of a spin network can be represented

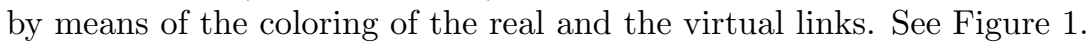

Vice versa, multiloop states can be decomposed in spin network states by simply symmetrizing along (real and virtual) nodes. This can be done par-

Living Reviews in Relativity (1998-1)

http://www.livingreviews.org 

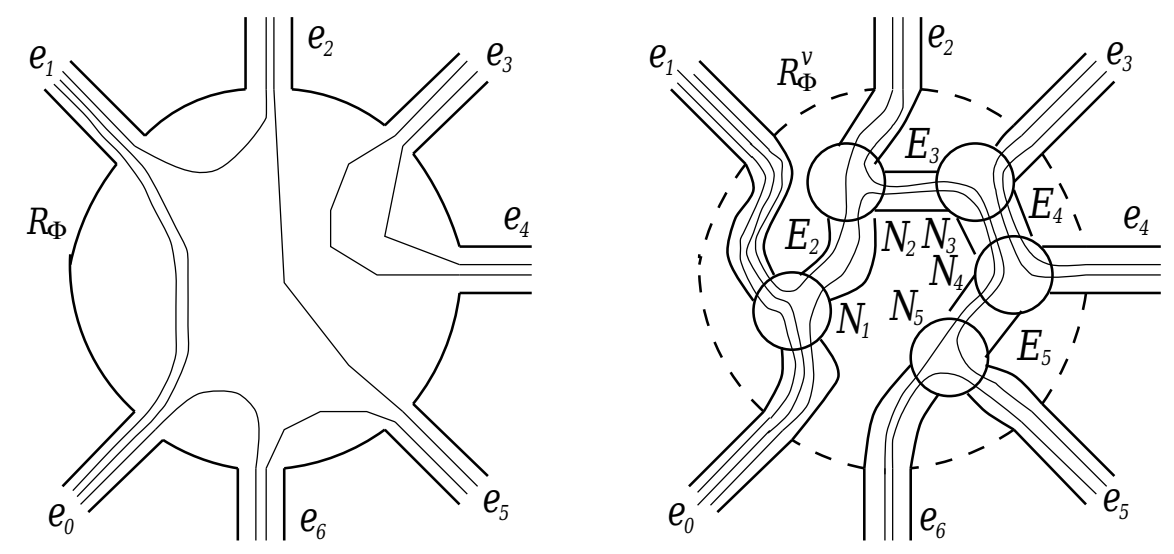

Figure 1: Construction of "virtual" nodes and "virtual" links over an $n$-valent node.

ticularly easily diagrammatically, as illustrated by the graphical formulae in [187, [7]]. These are standard formulae. In fact, it is well known that the tensor algebra of the $S U(2)$ irreducible representations admits a completely graphical notation. This graphical notation has been widely used for instance in nuclear and atomic physics. One can find it presented in detail in books such as [214, 5.3, 67] . The application of this diagrammatic calculus to quantum gravity is described in detail in [ [77], which I recommend to anybody who intends to perform concrete calculations in loop gravity.

It is interesting to notice that loop quantum gravity was first constructed in a pure diagrammatic notation, in [184]. The graphical nature of this calculus puzzled some, and the theory was accused of being vague and strange. Only later did researchers notice that the diagrammatic notation is actually very conventional $S U(2)$ diagrammatic calculus, well known in atomic and nuclear physics.]

\subsection{The representation}

I now define the quantum operators, corresponding to the $\mathcal{T}$-variables, as linear operators on $\mathcal{H}$. These form a representation of the loop variables Poisson algebra. The operator $\mathcal{T}[\alpha]$ acts diagonally

$$
\mathcal{T}[\alpha] \Psi(A)=-\operatorname{Tr} U_{\alpha}(A) \Psi(A) .
$$

\footnotetext{
${ }^{7}$ The following anecdote illustrates the level of the initial confusion. The first elaborate graphical computation of the eigenvalues of the area led to the mysterious formula $A_{p}=$ $\frac{1}{2} \hbar G \sqrt{p^{2}+2 p}$, with integer $p$. One day, Junichi Iwasaki, at the time a student, stormed into my office and told me to rewrite the formula in terms of the half integer $j=\frac{1}{2} p$. This yields the very familiar expression for the eigenvalues of the $S U(2)$ Casimir $A_{j}=\hbar G \sqrt{j(j+1)}$. Nobody had previously noticed this fact.
}

Living Reviews in Relativity (1998-1) http://www.livingreviews.org 
(Recall that products of loop states and spin network states are normalizable states). In diagrammatic notation, the operator simply adds a loop to a (linear combination of) multiloops

$$
\mathcal{T}[\alpha]|\Psi\rangle=|\alpha\rangle|\Psi\rangle .
$$

Higher order loop operators are expressed in terms of the elementary "grasp" operation. Consider first the operator $\mathcal{T}^{a}(s)[\alpha]$, with one hand in the point $\alpha(s)$. The operator annihilates all loop states that do not cross the point $\alpha(s)$. Acting on a loop state $|\beta\rangle$, it gives

$$
\mathcal{T}^{a}(s)[\alpha]|\beta\rangle=l_{0}^{2} \Delta^{a}[\beta, \alpha(s)]\left[|\alpha \# \beta\rangle-\left|\alpha \# \beta^{-1}\right\rangle\right],
$$

where we have introduced the elementary length $l_{0}$ by

$$
l_{0}^{2}=\hbar G=\frac{16 \pi \hbar G_{\text {Newton }}}{c^{3}}=16 \pi l_{\text {Planck }}^{2}
$$

and $\Delta^{a}$ and \# are defined in section 6.1 . This action extends by linearity, by continuity and by the Leibniz rule to products and linear combinations of loop states, and to the full $\mathcal{H}$. In particular, it is not difficult to compute its action on a spin network state [r7]. Higher order loop operators act similarly. It is a simple exercise to verify that these operators provide a representation of the classical Poisson loop algebra.

All the operators in the theory are then constructed in terms of these basic loop operators, in the same way in which in conventional QFT one constructs all operators, including the hamiltonian, in terms of creation and annihilation operators. The construction of the composite operators requires the development of regularization techniques that can be used in the absence of a background metric. These have been introduced in [[196] and developed in [186, [77, ㅍ. [24, ㅍ.37, 18].

\subsection{Algebraic version ("loop representation") and differ- ential version ("connection representation") of the for- malism, and their equivalence}

Imagine we want to quantize the one dimensional harmonic oscillator. We can consider the Hilbert space of square integrable functions $\psi(x)$ on the real line, and express the momentum and the hamiltonian as differential operators. Denote the eigenstates of the hamiltonian as $\psi_{n}(x)=\langle x \mid n\rangle$. It is well known that the theory can be expressed entirely in algebraic form in terms of the states $|n\rangle$. In doing so, all elementary operators are algebraic: $\hat{x}|n\rangle=$ $\frac{1}{\sqrt{2}}(|n-1\rangle+(n+1)|n+1\rangle), \hat{p}|n\rangle=\frac{-i}{\sqrt{2}}(|n-1\rangle-(n+1)|n+1\rangle)$. Similarly, in quantum gravity we can directly construct the quantum theory in the spinnetwork (or loop) basis, without ever mentioning functionals of the connections. This representation of the theory is denoted the "loop representation".

A section of the first paper on loop quantum gravity by Rovelli and Smolin [184] was devoted to a detailed study of "transformation theory" (in the sense of

Living Reviews in Relativity (1998-1)

http://www.livingreviews.org 
Dirac) on the state space of quantum gravity, and in particular on the relations between the loop states

$$
\psi(\alpha)=\langle\alpha \mid \psi\rangle
$$

and the states $\psi(A)$ giving the amplitude for a connection field configuration $A$, and defined by

$$
\psi(A)=\langle A \mid \psi\rangle .
$$

Here $|A\rangle$ are "eigenstates of the connection operator", or, more precisely (since the operator corresponding to the connection is ill defined in the theory) the generalized states that satisfy

$$
T[\alpha]|A\rangle=-\operatorname{Tr}\left[\mathcal{P} e^{\int_{\alpha} A}\right]|A\rangle .
$$

However, at the time of [184] the lack of a scalar product made transformation theory quite involved.

On the other hand, the introduction of the scalar product (14) gives a rigorous meaning to the loop transform. In fact, we can write, for every spin network $S$, and every state $\psi(A)$

$$
\psi(S)=\langle S \mid \psi\rangle=\left(\psi_{S}, \psi\right)
$$

This equation defines a unitary mapping between the two presentations of $\mathcal{H}$ : the "loop representation", in which one works in terms of the basis $|S\rangle$; and the "connection representation", in which one uses wave functionals $\psi(A)$.

The development of the connection representation followed a winding path through $C^{*}$-algebraic [13] and measure theoretical [15, [7, [16] methods. The work of Ashtekar, Isham, Lewandowski, Marolf, Mourao and Thiemann has finally put the connection representation on a firm ground, and, indirectly has much clarified the mathematics underlying the original loop approach. In the course of this development, doubts were raised about the precise relations between the connection and the loop formalisms. Today, the complete equivalence of these two approaches (always suspected) has been firmly established. In particular, the work of Roberto DePietri [ [76] has proven the unitary equivalence of the two formalisms. For a recent discussion see also [138].

\subsection{Other structures in $\mathcal{H}$}

The recent developments in the mathematical foundations of the connection representation have increased the mathematical rigor of the theory, raising it to the standards of mathematical physics. This has been obtained at the price of introducing heavy mathematical tools, often unfamiliar to the average physicist, perhaps widening the language gap between the quantum gravity and the high energy physics community.

The reason for searching a mathematical-physics level of precision is that in quantum gravity one moves on a very unfamiliar terrain -quantum field theory on manifolds- where the experience accumulated in conventional quantum field theory is often useless and sometimes even misleading. Given the unlikelihood

Living Reviews in Relativity (1998-1) http://www.livingreviews.org 
of finding direct experimental corroboration, the research can only aim, at least for the moment, at the goal of finding a consistent theory, with the correct limits in the regimes that we control experimentally. In these conditions, high mathematical rigor is the only assurance of the consistency of the theory. In the development of quantum field theory mathematical rigor could be very low because extremely accurate empirical verifications assured the physicists that "the theory may be mathematically meaningless, but it is nevertheless physical correct, and therefore the theory must make sense even if we do not understand how." Here, such an indirect experimental reassurance is lacking and the claim that the theory is well founded can be based only on a solid mathematical control of the theory.

One may object that a rigorous definition of quantum gravity is a vain hope, given that we do not even have a rigorous definition of QED, presumably a much simpler theory. The objection is particularly valid from the point of view of a physicist who views gravity "just as any other field theory like the ones we already understand". But the (serious) difficulties of QED and of the other conventional field theories are ultraviolet. The physical hope supporting the quantum gravity research program is that the ultraviolet structure of a diffeomorphism invariant quantum field theory is profoundly different from the one of conventional theories. Indeed, recall that in a very precise sense there is no short distance limit in the theory; the theory naturally cuts itself off at the Planck scale, due to the very quantum discreteness of spacetime. Thus the hope that quantum gravity could be defined rigorously may be optimistic, but it is not ill founded.

After these comments, let me briefly mention some of the structures that have been explored in $\mathcal{H}$. First of all, the spin network states satisfy the Kauffman axioms of the tangle theoretical version of recoupling theory [एँ29] (in the "classical" case $A=-1$ ) at all the points (in $3 \mathrm{~d}$ space) where they meet. (This fact is often misunderstood: recoupling theory lives in $2 \mathrm{~d}$ and is associated by Kauffman to knot theory by means of the usual projection of knots from $3 \mathrm{~d}$ to 2d. Here, the Kauffman axioms are not satisfied at the intersections created by the $2 \mathrm{~d}$ projection of the spin network, but only at the nodes in $3 \mathrm{~d}$. See [ [77] for a detailed discussion.) For instance, consider a 4-valent node of four links colored $a, b, c, d$. The color of the node is determined by expanding the 4 -valent node into a trivalent tree; in this case, we have a single internal links. The expansion can be done in different ways (by pairing links differently). These are related to each other by the recoupling theorem of pg. 60 in Ref. [I2.9]

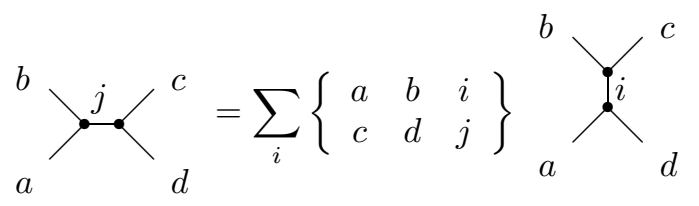

where the quantities $\left\{\begin{array}{lll}a & b & i \\ c & d & j\end{array}\right\}$ are $s u(2)$ six-j symbols (normalized as in [I29]). Equation (29) follows just from the definitions given above. Recoupling

Living Reviews in Relativity (1998-1)

http://www.livingreviews.org 
theory provides a powerful computational tool in this context.

Since spin network states satisfy recoupling theory, they form a TemperleyLieb algebra [12.9]. The scalar product (14) in $\mathcal{H}$ is given also by the TemperleyLieb trace of the spin networks, or, equivalently by the Kauffman brackets, or, equivalently, by the chromatic evaluation of the spin network. See Ref. [[7] for an extensive discussion of these relations.

Next, $\mathcal{H}$ admits a rigorous representation as an $L_{2}$ space, namely a space of square integrable functions. To obtain this representation, however, we have to extend the notion of connection, to a notion of "distributional connection". The space of the distributional connections is the closure of the space of smooth connection in a certain topology. Thus, distributional connections can be seen as limits of sequences of connections, in the same manner in which distributions can be seen as limits of sequences of functions. Usual distributions are defined as elements of the topological dual of certain spaces of functions. Here, there is no natural linear structure in the space of the connections, but there is a natural duality between connections and curves in $M$ : A smooth connection $A$ assigns a group element $U_{\gamma}(A)$ to every segment $\gamma$. The group elements satisfy certain properties. For instance if $\gamma$ is the composition of the two segments $\gamma_{1}$ and $\gamma_{2}$, then $U_{\gamma}(A)=U_{\gamma_{1}}(A) U_{\gamma_{2}}(A)$.

A generalized connection $\bar{A}$ is defined as a map that assigns an element of $S U(2)$, which we denote as $U_{\gamma}(\bar{A})$ or $\bar{A}(\gamma)$, to each (oriented) curve $\gamma$ in $M$, satisfying the following requirements: i) $\bar{A}\left(\gamma^{-1}\right)=(\bar{A}(\gamma))^{-1}$; and, ii) $\bar{A}\left(\gamma_{2} \circ\right.$ $\left.\gamma_{1}\right)=\bar{A}\left(\gamma_{2}\right) \cdot \bar{A}\left(\gamma_{1}\right)$, where $\gamma^{-1}$ is obtained from $\gamma$ by reversing its orientation, $\gamma_{2} \circ \gamma_{1}$ denotes the composition of the two curves (obtained by connecting the end of $\gamma_{1}$ with the beginning of $\left.\gamma_{2}\right)$ and $\bar{A}\left(\gamma_{2}\right) \cdot \bar{A}\left(\gamma_{1}\right)$ is the composition in $S U(2)$. The space of such generalized connections is denoted $\overline{\mathcal{A}}$. The cylindrical functions $\Psi_{\Gamma, f}(A)$, defined in section 6.3 as functions on the space of smooth connections, extend immediately to generalized connections

$$
\Psi_{\Gamma, f}(\bar{A})=f\left(\bar{A}\left(\gamma_{1}, \ldots, \bar{A}\left(\gamma_{n}\right)\right) .\right.
$$

We can define a measure $d \mu_{0}$ on the space of generalized connections $\overline{\mathcal{A}}$ by

$$
\int d \mu_{0}[\bar{A}] \Psi_{\Gamma, f}(\bar{A}) \equiv \int_{S U(2)^{n}} d g_{1} \ldots d g_{n} f\left(g_{1} \ldots g_{n}\right) .
$$

In fact, one may show that (31) defines (by linearity and continuity) a welldefined absolutely continuous measure on $\overline{\mathcal{A}}$. This is the Ashtekar-Lewandowski (or Ashtekar-Lewandowski-Baez) measure [15, ㅌ, 피, 31]. Then, one can prove that $\mathcal{H}=L_{2}\left[\overline{\mathcal{A}}, d \mu_{0}\right]$, under the natural isomorphism given by identifying cylindrical functions. It follows immediately that the transformation (19) between the connection representation and the "old" loop representation is given by

$$
\psi(\alpha)=\int d \mu_{0}[\bar{A}] \operatorname{Tr} \overline{\mathcal{P e}^{\int_{\alpha} \mathrm{A}}} \Psi(\overline{\mathrm{A}}) .
$$

This is the loop transform formula that was derived heuristically in [184]; here it becomes rigorously defined.

Living Reviews in Relativity (1998-1) http://www.livingreviews.org 
Furthermore, $\mathcal{H}$ can be seen as the projective limit of the projective family of the Hilbert spaces $\mathcal{H}_{\Gamma}$, associated to each graph $\Gamma$ immersed in $M . \mathcal{H}_{\Gamma}$ is defined as the space $L_{2}\left[S U(2)^{n}, d g_{1} \ldots d g_{n}\right]$, where $n$ is the number of links in $\Gamma$. The cylindrical function $\Psi_{\Gamma, f}(A)$ is naturally associated to the function $f$ in $\mathcal{H}_{\Gamma}$, and the projective structure is given by the natural map (13) [119, 148].

Finally, Ashtekar and Isham [113] have recovered the representation of the loop algebra by using $\mathrm{C}^{*}$-algebra representation theory: The space $\overline{\mathcal{A}} / \mathcal{G}$, where $\mathcal{G}$ is the group of local $S U(2)$ transformations (which acts in the obvious way on generalized connections), is precisely the Gelfand spectrum of the abelian part of the loop algebra. One can show that this is a suitable norm closure of the space of smooth $S U(2)$ connections over physical space, modulo gauge transformations.

Thus, a number of powerful mathematical tools are at hand for dealing with nonperturbative quantum gravity. These include: Penrose's spin network theory, $S U(2)$ representation theory, Kauffman tangle theoretical recoupling theory, Temperley-Lieb algebras, Gelfand's $C^{*}$ algebra spectral representation theory, infinite dimensional measure theory and differential geometry over infinite dimensional spaces.

\subsection{Diffeomorphism invariance}

The next step in the construction of the theory is to factor away diffeomorphism invariance. This is a key step for two reasons. First of all, $\mathcal{H}$ is a "huge" non-separable space. It is far "too large" for a quantum field theory. However, most of this redundancy is gauge, and disappears when one solves the diffeomorphism constraint, defining the diff-invariant Hilbert space $\mathcal{H}_{\text {Diff }}$. This is the reason for which the loop representation, as defined here, is of great value in diffeomorphism invariant theories only.

The second reason is that $\mathcal{H}_{\text {Diff }}$ turns out to have a natural basis labeled by knots. More precisely by "s-knots". An s-knot $s$ is an equivalence class of spin networks $S$ under diffeomorphisms. An s-knot is characterized by its "abstract" graph (defined only by the adjacency relations between links and nodes), by the coloring, and by its knotting and linking properties, as in knot-theory. Thus, the physical quantum states of the gravitational field turn out to be essentially classified by knot theory.

There are various equivalent ways of obtaining $\mathcal{H}_{\text {Diff }}$ from $\mathcal{H}$. One can use regularization techniques for defining the quantum operator corresponding to the classical diffeomorphism constraint in terms of elementary loop operators, and then find the kernel of such operator. Equivalently, one can factor $\mathcal{H}$ by the natural action of the diffeomorphism group that it carries. Namely

$$
\mathcal{H}_{\text {Diff }}=\frac{\mathcal{H}}{\operatorname{Diff}(M)} \text {. }
$$

There are several rigorous ways for defining the quotient of a Hilbert space by the unitary action of a group. See in particular the construction in [II]], which follows the ideas of Marolf and Higuchi [144, 146, 1147, 1077].

Living Reviews in Relativity (1998-1)

http://www.livingreviews.org 
In the quantum gravity literature, a big deal has been made of the problem that a scalar product is not defined on the space of solutions of a constraint $\hat{C}$, defined on a Hilbert space $\mathcal{H}$. This, however, is a false problem. It is true that if zero is in the continuum spectrum of $\hat{C}$, then the corresponding eigenstates are generalized states and the $\mathcal{H}$ scalar product is not defined between them. But the generalized eigenspaces of $\hat{C}$, including the kernel, inherit nevertheless a scalar product from $\hat{H}$. This can be seen in a variety of equivalent ways. For instance, it can be seen from the following theorem. If $\hat{C}$ is self adjoint, then there exist a measure $\mu(\lambda)$ on its spectrum and a family of Hilbert spaces $\mathcal{H}(\lambda)$ such that

$$
\mathcal{H}=\int d \mu(\lambda) \mathcal{H}(\lambda)
$$

where the integral is the continuous sum of Hilbert spaces described, for instance, in [100]. Clearly $\mathcal{H}(0)$ is the kernel of $\hat{C}$ equipped with a scalar product. This is discussed, for instance, in [162].

There are two distinct possibilities for factoring away the diffeomorphisms in the quantum theory, yielding two distinct version of the theory. The first possibility is to factor away smooth transformations of the manifold. In doing so, finite dimensional moduli spaces associated with high valence nodes appear [98], so that the resulting Hilbert space is still non-separable. The physical relevance of these moduli parameters is unclear at this stage, since they do not seem to play any role in the quantum theory. Alternatively, one can consistently factor away continuous transformations of the manifold. This possibility has been explored by Zapata in [215, [216], and seems to lead to a consistent theory free of the residual non separability.

\subsection{Dynamics}

Finally, the definition of the theory is completed by giving the hamiltonian constraint. A number of approaches to the definition of a hamiltonian constraint have been attempted in the past, with various degrees of success. Recently, however, Thiemann has succeeded in providing a regularization of the hamiltonian constraint that yields a well defined, finite operator. Thiemann's construction [2018, [202, 2013] is based on several clever ideas. I will not describe it here. Rather, I will sketch below the final form of the constraint (for the Lapse=1 case), following [T75].

I begin with the Euclidean hamiltonian constraint. We have

$$
\hat{H}|s\rangle=\sum_{i} \sum_{(I J)} \sum_{\epsilon= \pm 1} \sum_{\epsilon^{\prime}= \pm 1} A_{\epsilon \epsilon^{\prime}}\left(p_{i} \ldots p_{n}\right) \hat{D}_{i ;(I J), \epsilon \epsilon^{\prime}}|s\rangle .
$$

Here $i$ labels the nodes of the s-knot $s ;(I J)$ labels couples of (distinct) links emerging from $i . p_{1} \ldots p_{n}$ are the colors of the links emerging from $i . \hat{D}_{i ;(I J) \epsilon \epsilon^{\prime}}$ is the operator that acts on an $s$-knot by: (i) creating two additional nodes, one along each of the two links $I$ and $J$; (ii) creating a novel link, colored 1 , joining these two nodes, (iii) assigning the coloring $p_{I}+\epsilon$ and, respectively, $p_{J}+\epsilon^{\prime}$ to

Living Reviews in Relativity (1998-1) http://www.livingreviews.org 


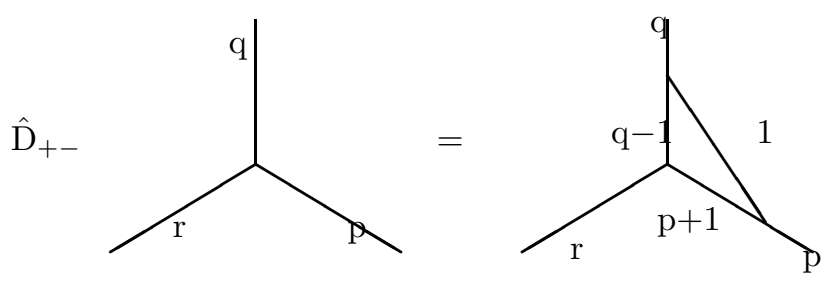

Figure 2: Action of $\hat{D}_{i ;(I J) \epsilon \epsilon^{\prime}}$.

the links that join the new formed nodes with the node $i$. This is illustrated in Figure 2.

The coefficients $A_{\epsilon \epsilon^{\prime}}\left(p_{i} \ldots p_{n}\right)$, which are finite, can be expressed explicitly (but in a rather laborious way) in terms of products of linear combinations of $6-j$ symbols of $S U(2)$, following the techniques developed in detail in [r7]. Some of these coefficients have been explicitly computed [52]. The Lorentzian hamiltonian constraint is given by a similar expression, but quadratic in the $\hat{D}$ operators.

The operator defined above is obtained by introducing a regularized expression for the classical hamiltonian constraint, written in terms of elementary loop observables, turning these observables into the corresponding operators and taking the limit. The construction works rather magically, relying on the fact, first noticed in [188], that certain operator limits $\hat{O}_{\epsilon} \rightarrow \hat{O}$ turn out to be finite on diff invariant states, thanks to the fact that, for $\epsilon$ and $\epsilon^{\prime}$, sufficiently small, $\hat{O}_{\epsilon}|\Psi\rangle$ and $\hat{O}_{\epsilon^{\prime}}|\Psi\rangle$ are diffeomorphic equivalent. Thus, here diff invariance plays again a crucial role in the theory.

For a discussion of the problems raised by the Thiemann operator and of the variant proposed, see section 8 .

\subsection{Unfreezing the frozen time formalism: the covariant form of loop quantum gravity}

A recent development in the formalism is the translation of loop quantum gravity into spacetime covariant form. This was initiated in [181, [160] by following the steps that Feynman took in defining path integral quantum mechanics starting from the Schrödinger canonical theory. More precisely, it was proven in [I6T] that the matrix elements of the operator $U(T)$

$$
U(T) \equiv e^{\int_{0}^{T} d t \int d^{3} x \hat{H}(x)},
$$

obtained exponentiating the (Euclidean) hamiltonian constraint in the proper time gauge (the operator that generates evolution in proper time) can be expanded in a Feynman sum over paths. In conventional QFT each term of a Feynman sum corresponds naturally to a certain Feynman diagram, namely a

Living Reviews in Relativity (1998-1)

http://www.livingreviews.org 
set of lines in spacetime meeting at vertices (branching points). A similar natural structure of the terms appears in quantum gravity, but surprisingly the diagrams are now given by surfaces is spacetime that branch at vertices. Thus, one has a formulation of quantum gravity as a sum over surfaces in spacetime. Reisenberger [158] and Baez [33] have argued in the past that such a formulation should exist, and Iwasaki has developed a similar construction in $2+1$ dimensions. Intuitively, the time evolution of a spin network in spacetime is given by a colored surface. The surfaces capture the gravitational degrees of freedom. The formulation is "topological" in the sense that one must sum over topologically inequivalent surfaces only, and the contribution of each surface depends on its topology only. This contribution is given by the product of elementary "vertices", namely points where the surface branches.

The transition amplitude between two s-knot states $\left|s_{i}\right\rangle$ and $\left|s_{f}\right\rangle$ in a proper time $T$ is given by summing over all (branching, colored) surfaces $\sigma$ that are bounded by the two s-knots $s_{i}$ and $s_{f}$

$$
\left\langle s_{f}|U(T)| s_{i}\right\rangle=\sum_{\substack{\sigma \\ \partial \sigma=s_{i} \cup s_{f}}} \mathcal{A}[\sigma](T) .
$$

The weight $\mathcal{A}[\sigma](T)$ of the surface $\sigma$ is given by a product over the $n$ vertices $v$ of $\sigma$ :

$$
\mathcal{A}[\sigma](T)=\frac{(-i T)^{n}}{n !} \prod_{v} A_{v}(\sigma) .
$$

The contribution $A_{v}(\sigma)$ of each vertex is given by the matrix elements of the hamiltonian constraint operator between the two s-knots obtained by slicing $\sigma$ immediately below and immediately above the vertex. They turn out to depend only on the colors of the surface components immediately adjacent the vertex $v$. The sum turns out to be finite and explicitly computable order by order.

As in the usual Feynman diagrams, the vertices describe the elementary interactions of the theory. In particular, here one sees that the complicated structure of the Thiemann hamiltonian, which makes a node split into three nodes, corresponds to a geometrically very simple vertex. Figure 3 is a picture of the elementary vertex. Notice that it represents nothing but the spacetime evolution of the elementary action of the hamiltonian constraint, given in Figure [2].

An example of a surface in the sum is given in Figure 4 .

The sum over surfaces version of loop quantum gravity provides a link with certain topological quantum field theories and in particular with the the CraneYetter model [7], [72, 7.3, 74, 75], which admit an extremely similar representation. For a discussion on the precise relation between topological quantum field theory and diffeomorphism invariant quantum field theory, see [100] and [171, 12:3, 8:3].

The idea of expressing the theory as a sum over surfaces has been developed by Baez [26], who has studied the general form of generally covariant quantum field theories formulated in this manner, and by Smolin and Markopoulou [143],

Living Reviews in Relativity (1998-1) http://www.livingreviews.org 


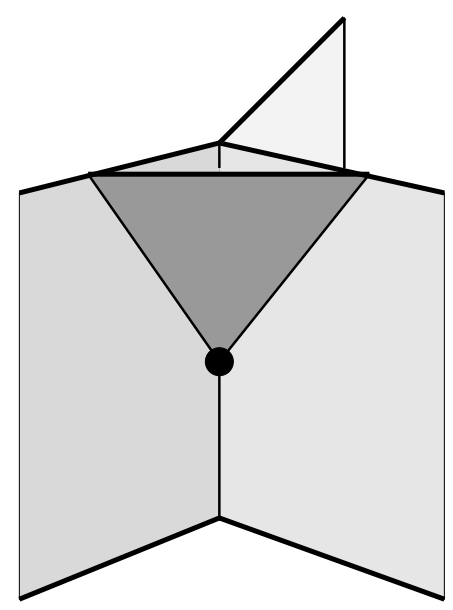

Figure 3: The elementary vertex.

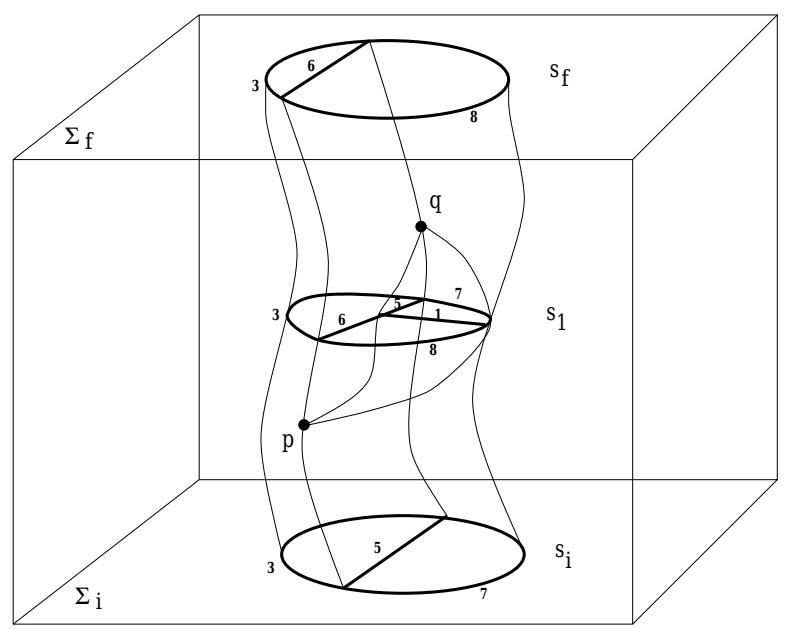

Figure 4: A term of second order.

Living Reviews in Relativity (1998-1)

http://www. livingreviews.org 
who have studied how to directly capture the Lorentzian causal structure of general relativity modifying the elementary vertices. They have also explored the idea that the long range correlations of the low energy regime of the theory are related to the existence of a phase transition in the microscopic dynamics, and have found intriguing connections with the theoretical description of percolation.

Living Reviews in Relativity (1998-1) http://www.livingreviews.org 


\section{Physical Results}

In section 6, I have sketched the basic structure of loop quantum gravity. This structure has been developed in a number of directions, and has been used to derive a number of results. Without any ambition of completeness, I list below some of these developments.

\subsection{Technical}

- Solutions of the hamiltonian constraints. One of the most surprising results of the theory is that it has been possible to find exact solutions of the hamiltonian constraint. This follows from the key result that the action of the hamiltonian constraints is non vanishing only over nodes of the s-knots [183, 184]. Therefore s-knots without nodes are physical states that solve the quantum Einstein dynamics. There is an infinite number of independent states of this sort, classified by conventional knot theory. The physical interpretation of these solutions is still rather obscure. Various other solutions have been found. See the recent review [ $[82]$ and references

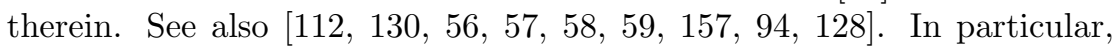
Pullin has studied in detail solutions related to the Chern-Simon term in the connection representation and to the Jones polynomial in the loop representation. According to a celebrated result by Witten [2]2], the two are the loop transform of each other.

- Time evolution. Strong field perturbation expansion. "Topological Feynman rules". Trying to describe the temporal evolution of the quantum gravitational field by solving the hamiltonian constraint yields the conceptually well-defined [168], but notoriously nontransparent, frozen-time formalism. An alternative is to study the evolution of the gravitational degrees of freedom with respect to some matter variable, coupled to the theory, which plays the role of a phenomenological "clock". This approach has lead to the tentative definition of a physical hamiltonian [188, 4.9], and to a preliminary investigation of the possibility of transition amplitudes between s-knot states, order by order in a (strong coupling) perturbative expansion [[75]. In this context, diffeomorphism invariance, combined with the key result that the hamiltonian constraint acts on nodes only, implies that the "Feynman rules" of such an expansion are purely topological and combinatorial.

- Fermions. Fermions have been added to the theory [1449, 1550, 36, 2044]. Remarkably, all the important results of the pure GR case survive in the $\mathrm{GR}+$ fermions theory. Not surprisingly, fermions can be described as open ends of "open spin networks".

- Maxwell and Yang-Mills. The extension of the theory to the Maxwell field has been studied in [[32, M1]. The extension to Yang-Mills theory has been explored recently in [201]]. In [20I]], Thiemann shows that the

Living Reviews in Relativity (1998-1)

http://www.livingreviews.org 
Yang-Mills term in the quantum hamiltonian constraint can be defined in a rigorous manner, extending the methods of [208, 202, 2013]. A remarkable result in this context is that ultraviolet divergences do not seem to appear, strongly supporting the expectation that the natural cut off introduced by quantum gravity might cure the ultraviolet difficulties of conventional quantum field theory.

- Application to other theories. The loop representation has been applied in various other contexts such as $2+1$ gravity [14, 145, 2I] (on $2+1$ quantum gravity, in the loop and in other representations, see [63]) and others [2:2].

- Lattice and simplicial models. A number of interesting discretized versions of the theory are being studied. See in particular [140, [159, , 14, [179].

\subsection{Physical}

\section{- Planck scale discreteness of space.}

The most remarkable physical result obtained from loop quantum gravity is, in my opinion, evidence for a physical (quantum) discreteness of space at the Planck scale. This is manifested in the fact that certain operators corresponding to the measurement of geometrical quantities, in particular area and volume, have discrete spectrum. According to the standard interpretation of quantum mechanics (which we adopt), this means that the theory predicts that a physical measurement of an area or a volume will necessarily yield quantized results. Since the smallest eigenvalues are of Planck scale, this implies that there is no way of observing areas or volumes smaller than Planck scale. Space comes in "quanta" in the same manner as the energy of an oscillator. The spectra of the area and volume operators have been computed with much detail in loop quantum gravity. These spectra have a complicated structure, and they constitute detailed quantitative physical predictions of loop quantum gravity on Planck scale physics. If we had experimental access to Planck scale physics, they would allow the theory to be empirically tested in great detail.

A few comments are in order.

- The result of the discreteness of area and volume is due to Rovelli and Smolin, and appeared first in reference [I86]. Later, the result has been recovered by alternative techniques and extended by a number of authors. In particular, Ashtekar and Lewandowski [18] have repeated the derivation, using the connection representation, and have completed the computation of the spectrum (adding the sector which was not computed in [186].) The Ashtekar-Lewandowski component of the spectrum has then been rederived in the loop representation by Frittelli Lehner and Rovelli in [84]. Loll has employed lattice techniques to point out a numerical error in [1866] (corrected in the Erratum) in the eigenvalues of the volume. The analysis of the volume

Living Reviews in Relativity (1998-1) http://www.livingreviews.org 
eigenvalues has been performed in [77], where general techniques for performing these calculations are described in detail. The spectrum of the volume has then been analyzed also in [20.5]. There are also a few papers that have anticipated the main result presented in [186]. In particular, Ashtekar Rovelli and Smolin have argued for a physical discreteness of space emerging from the loop representation in [24], where some of the eigenvalues of the area already appear, although in implicit form. The first explicit claim that area eigenvalues might in principle be observable (in the presence of matter) is by Rovelli in [17.2].

- The reader will wonder why area and volume seem to play here a role more central than length, when classical geometry is usually described in terms of lengths. The reason is that the length operator is difficult to define and of more difficult physical interpretations. For attempts in this direction, see [206]. Whether this is simply a technical difficulty, or it reflects some deep fact, is not clear to me. 8

- Area and volume are not gauge invariant operators. Therefore, we cannot directly interpret them as representing physical measurements. Realistic physical measurements of areas and volumes always refer to physical surfaces and spatial regions, namely surfaces and spatial regions determined by some physical object. For instance, I can measure the area of the surface of a certain table. In the dynamical theory that describes the gravitational field as well as the table, the area of the surface of the table is a diffeomorphism invariant quantity $A$, which depends on gravitational as well as matter variables. In the quantum theory, $A$ will be represented by a diffeomorphism invariant operator. Now, as first realized in [172], it is plausible that the operator $A$ is, mathematically, the same operator as the pure gravity area operator. This is because we can gauge fix the matter variables, and use matter location as coordinates, so that non-diff-invariant observables in the pure gravity theory correspond precisely to diff-invariant observables in the matter+gravity theory. Thus, discreteness of the spectrum of the area operator is likely to imply discreteness of physically measurable areas, but it is important to emphasize that this implication is based on some additional hypothesis on the relation between the pure gravity and the gravity+matter theories.

The discreteness of area and volume is derived as follows. Consider the area $A$ of a surface $\Sigma$. The physical area $A$ of $\Sigma$ depends on the metric,

\footnotetext{
${ }^{8} \mathrm{I}$ include here a comment on this issue I received from John Baez: "I believe there is a deep reason why area is more fundamental than length in loop quantum gravity. One way to say it is that the basic field is not the tetrad $e$ but the 2-form $E=e \wedge e$. Another way to say it is that the loop representation is based on the theory of quantized angular momentum. Angular momentum is not a vector but a bivector, so it corresponds not to an arrow but to an oriented area element." On this, see Baez's [루]. On the relation between $E$ field and area see in particular [ए]त].
}

Living Reviews in Relativity (1998-1)

http://www.livingreviews.org 
namely on the gravitational field. In a quantum theory of gravity, the gravitational field is a quantum field operator, and therefore we must describe the area of $\Sigma$ in terms of a quantum observable, described by an operator $\hat{A}$. We now ask what the quantum operator $\hat{A}$ in nonperturbative quantum gravity is. The result can easily be worked out by writing the standard formula for the area of a surface, and replacing the metric with the appropriate function of the loop variables. Promoting these loop variables to operators, we obtain the area operator $\hat{A}$. The actual construction of this operator requires regularizing the classical expression and then taking the limit of a sequence of operators, in a suitable operator topology. For the details of this construction, see [186, 77, 84, 52]. An alternative regularization technique is discussed in [18]. The resulting area operator $\hat{A}$ acts as follows on a spin network state $|S\rangle$ (assuming here for simplicity that $S$ is a spin network without nodes on $\Sigma$ ):

$$
\hat{A}[\Sigma]|S\rangle=\left(\frac{l_{0}^{2}}{2} \sum_{i \in\{S \cap \Sigma\}} \sqrt{p_{i}\left(p_{i}+2\right)}\right)|S\rangle
$$

where $i$ labels the intersections between the spin network $S$ and the surface $\Sigma$, and $p_{i}$ is the color of the link of $S$ crossing the $i-t h$ intersection.

This result shows that the spin network states (with a finite number of intersection points with the surface and no nodes on the surface) are eigenstates of the area operator. The corresponding spectrum is labeled by multiplets $\vec{p}=\left(p_{1}, \ldots, p_{n}\right)$ of positive half integers, with arbitrary $n$, and given by

$$
A_{\vec{p}}[\Sigma]=\frac{l_{0}^{2}}{2} \sum_{i} \sqrt{p_{i}\left(p_{i}+2\right)} .
$$

Shifting from color to spin notation reveals the $S U(2)$ origin of the spectrum:

$$
A_{\vec{j}}[\Sigma]=l_{0}^{2} \sum_{i} \sqrt{j_{i}\left(j_{i}+1\right)},
$$

where $j_{1}, \ldots, j_{n}$ are half integers. For the full spectrum, see [I8] (connection representation) and [84] (loop representation).

A similar result can be obtained for the volume [186, 141, 142, [77, 1138]. Let us restrict ourselves here, for simplicity, to spin networks $S$ with nondegenerate four-valent nodes, labeled by an index $i$. Let $a_{i}, b_{i}, c_{i}, d_{i}$ be the colors of the links adjacent to the $i-t h$ node and let $J_{i}$ label the basis in the intertwiner space. The volume operator $\hat{V}$ acts as follows

$$
\hat{V}|S\rangle=\frac{l_{0}^{3}}{2} \sum_{i} \sqrt{\left|i W^{(i)}\left(a_{i}, b_{i}, c_{i}, d_{i}\right)\right|}|S\rangle
$$

where $W^{i}$ is an operator that acts on the finite dimensional space of the intertwiners in the $i-t h$ node, and its matrix elements are explicitly given

Living Reviews in Relativity (1998-1) http://www.livingreviews.org 
(in a suitable basis) by $(\epsilon= \pm$ )

$$
\begin{aligned}
& W^{i}(a, b, c, d)_{t-\epsilon}^{t+\epsilon}=-\epsilon(-1)^{\frac{a+b+c+d}{2}} \times \\
& {\left[\frac{1}{4 t(t+2)} \frac{a+b+t+3}{2} \frac{c+d+t+3}{2}\right.} \\
& \frac{1+a+b-t}{2} \frac{1+a+t-b}{2} \frac{1+b+t-a}{2} \\
& \left.\quad \frac{1+c+d-t}{2} \frac{1+c+t-d}{2} \frac{1+d+t-c}{2}\right]^{\frac{1}{2}} .
\end{aligned}
$$

See [52]. The volume eigenvalues $v_{i}$ are obtained by diagonalizing these matrices. For instance, in the simple case $a=b, c=d=1$, we have

$$
v_{a}=\frac{(\hbar G)^{3}}{2} \sqrt{a(a+2)}
$$

if $d=a+b+c$, we have

$$
v_{a, b, c}=\frac{(\hbar G)^{3}}{2} \sqrt{a b c(a+b+c)} .
$$

For more details, and the full derivation of these formulas, see [52, 20.5]

- Classical limit. Quantum states representing flat spacetime. Weaves. Discrete small scale structure of space.

The s-knot states do not represent excitations of the quantum gravitational field over flat space, but rather over "no-space", or over the $g_{\mu \nu}=0$ solution. A natural problem is then how flat space (or any other smooth geometry) might emerge from the theory. Notice that in a general relativistic context the Minkowski solution does not have all the properties of the conventional field theoretical vacuum. (In gravitational physics there is no real equivalent of the conventional vacuum, particularly in the spatially compact case.) One then expects that flat space is represented by some highly excited state in the theory. States in $\mathcal{H}$ that describe flat space when probed at low energy (large distance) have been studied in [24, 217, 50, 99]. These have a discrete structure at the Planck scale. Furthermore, small excitations around such states have been considered in [124], where it is shown that $\mathcal{H}$ contains all "free graviton" physics, in a suitable approximation.

\section{- The Bekenstein-Mukhanov effect.}

Recently, Bekenstein and Mukhanov [47] have suggested that the thermal nature of Hawking's radiation [104, 10.5] may be affected by quantum properties of gravity (For a review of earlier suggestions in this direction, see [1.93]). Bekenstein and Mukhanov observe that in most approaches to quantum gravity the area can take only quantized values [97]. Since the area of the black hole surface is connected to the black hole mass,

Living Reviews in Relativity (1998-1)

http://www.livingreviews.org 
black hole mass is likely to be quantized as well. The mass of the black hole decreases when radiation is emitted. Therefore emission happens when the black hole makes a quantum leap from one quantized value of the mass (energy) to a lower quantized value, very much as atoms do. A consequence of this picture is that radiation is emitted at quantized frequencies, corresponding to the differences between energy levels. Thus, quantum gravity implies a discretized emission spectrum for the black hole radiation.

This result is not physically in contradiction with Hawking's prediction of a continuous thermal spectrum, because spectral lines can be very dense in macroscopic regimes. But Bekenstein and Mukhanov observed that if we pick the simplest ansatz for the quantization of the area -that the Area is quantized in multiple integers of an elementary area $A_{0^{-}}$, then the emitted spectrum turns out to be macroscopically discrete, and therefore very different from Hawking's prediction. I will denote this effect as the kinematical Bekenstein-Mukhanov effect. Unfortunately, however, the kinematical Bekenstein-Mukhanov effect disappears if we replace the naive ansatz with the spectrum (41) computed from loop quantum gravity. In loop quantum gravity, the eigenvalues of the area become exponentially dense for a macroscopic black hole, and therefore the emission spectrum can be consistent with Hawking's thermal spectrum. This is due to the details of the spectrum (41) of the area. A detailed discussion of this result is in [44], but the result was already contained (implicitly, in the first version) in [[18]. It is important to notice that the density of the eigenvalues shows only that the simple kinematical argument of Bekenstein and Mukhanov is not valid in this theory, and not that their conclusions is necessarily wrong. As emphasized by Mukhanov, a discretization of the emitted spectrum could still be originated dynamically.

\section{- Black Hole Entropy from Loop Quantum Gravity.}

Indirect arguments [104, 105, 46, 2010] strongly support the idea that a Schwarzschild black hole of (macroscopic) area $A$ behaves as a thermodynamical system governed by the Bekenstein-Hawking entropy

$$
S=\frac{k}{4 \hbar G_{\text {Newton }}} A
$$

( $k$ is the Boltzmann constant; here I put the speed of light equal to one, but write the Planck and Newton constants explicitly). A physical understanding and a first principles derivation of this relation require quantum gravity, and therefore represent a challenge for every candidate theory of quantum theory. A derivation of the Bekenstein-Hawking expression (46) for the entropy of a Schwarzschild black hole of surface area $A$ via a statistical mechanical computation, using loop quantum gravity, was obtained in [133., ए3.34, ए76].

This derivation is based on the ideas that the entropy of the hole originates from the microstates of the horizon that correspond to a given

Living Reviews in Relativity (1998-1)

http://www.livingreviews.org 
macroscopic configuration [213, 65, 64, 38, 3.9]. Physical arguments indicate that the entropy of such a system is determined by an ensemble of configurations of the horizon with fixed area [ए76]. In the quantum theory these states are finite in number, and can be counted [1333, 1.34]. Counting these microstates using loop quantum gravity yields

$$
S=\frac{c}{\gamma} \frac{k}{4 \hbar G_{\text {Newton }}} A .
$$

(An alternative derivation of this result has been announced from Ashtekar, Baez, Corichi and Krasnov [ए2].) $\gamma$ is defined in section 6, and $c$ is a real number of the order of unity that emerges from the combinatorial calculation (roughly, $c \sim 1 / 4 \pi$ ). If we choose $\gamma=c$, we get (46) [189, [1]]. Thus, the theory is compatible with the numerical constant in the BekensteinHawking formula, but does not lead to it univocally. The precise significance of this fact is under discussion. In particular, the meaning of $\gamma$ is unclear. Jacobson has suggested [126] that finite renormalization effects may affect the relation between the bare and the effective Newton constant, and this may be reflected in $\gamma$. For discussion of the role of $\gamma$ in the theory, see [18.9]. On the issue of entropy in loop gravity, see also [194].

Living Reviews in Relativity (1998-1)

http://www.livingreviews.org 


\section{Main Open Problems and Main Current Lines of Investigation}

Hamiltonian constraint. The kinematics of the theory is well understood, both physically (quanta of area and volume, polymer-like geometry) and from the mathematical point of view $(\mathcal{H}$, s-knot states, area and volume operators). The part of the theory which is not yet fully under control is the dynamics, which is determined by the hamiltonian constraint. A plausible candidate for the quantum hamiltonian constraint is the operator introduced by Thiemann [208, [202, [203]. The commutators of the Thiemann operator with itself and with the diffeomorphism constraints close, and therefore the operator defines a complete and consistent quantum theory. However, doubts have been raised on the physical correctness of this theory, and some variants of the operator have been considered.

The doubts originate from various considerations. First, Lewandowski, Marolf and others have stressed the fact the quantum constraint algebra closes, but it is not isomorphic to the classical constraint algebra of GR [139]. Recently, a detailed analysis of this problem has been completed by Marolf, Lewandowski, Gambini and Pullin [90]]. The failure to reproduce the classical constraint algebra has been disputed, and is not necessarily a problem, since the only strict requirement on the quantum theory, besides consistency, is that its gauge invariant physical predictions match the ones of classical general relativity in the appropriate limit. Still, the difference in the algebras may be seen as circumstantial evidence (not a proof) for the failure of the classical limit. The issue is technically delicate and still controversial. I hope I will be able to say something more definitive in the next update of this review.

Second, Brügmann [54] and Smolin [1192] have pointed out a sort of excess "locality" in the form of the operator, which, intuitively, seems in contradiction with the propagation properties of the Einstein equations. Finally, by translating the Thiemann operator into a spacetime covariant four-dimensional formalism, Reisenberger and Rovelli have noticed a suspicious lack of manifest 4-d covariance in the action of the operator [160], a fact pointing again to the possibility of anomalies in the quantum constraint algebra.

Motivated by these doubts, several variants of Thiemann's operator have been suggested. The original Thiemann's operators is constructed using the volume operator. There are two versions of the volume operator in the literature: $V_{R L}$, introduced in [186] and $V_{A L}$, introduced in [15, 17, 16]. See [138] for a detailed comparison. Originally, Thiemann thought that using $V_{R S}$ in the hamiltonian constraint would yield difficulties, but it later became clear that this is not the case [13.9]. Both versions of the volume can be used in the definition, yielding two alternative versions of the hamiltonian [13.9]. Next, in its simplest version the operator is non-symmetric.

Living Reviews in Relativity (1998-1) http://www.livingreviews.org 


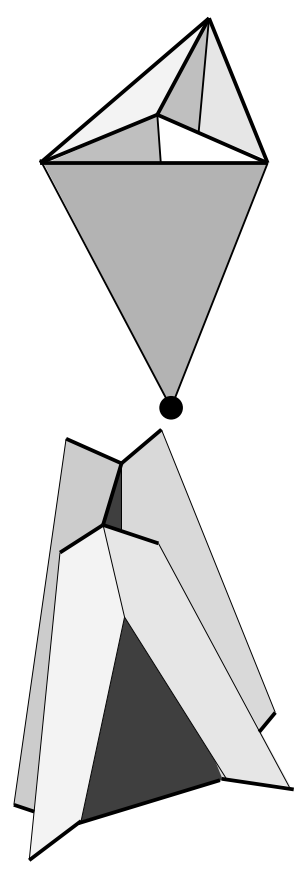

Figure 5: The crossing symmetric vertices.

Since the classical hamiltonian constraint is real (on $S U(2)$ gauge invariant states), one might expect a corresponding self-adjoint quantum operator. If Accordingly, several ways of symmetrizing the operator have been considered (see a list in [1.3.9]). Next, Smolin has considered some ad hoc modifications of the constraint in [[T2]. Finally, the spacetime covariant formalism in [160] naturally suggest a "covariantisation" of the operator, described in [1160] under the name of "crossing symmetry". This covariantisation amounts to adding to the vertex described in Figure [3] the vertices, described in Figure 5, which are simply obtained by re-orienting Figure 3 in spacetime.

A full comparative analysis of this various proposals would be of great interest.

Ultimately, the final tests of any proposal for the hamiltonian constraint operator must be consistency and a correct classical limit. Thus, the solution of the hamiltonian constraint puzzle is likely to be subordinate

\footnotetext{
${ }^{9}$ There is an argument which is often put forward against the requirement of self-adjointness of the hamiltonian constraint $H$ : Let $H$ be self-adjoint and $O$ be any operator of the form $O=[H, A]$, where $A$ is any operator (many operators that we do not expect could vanish have this form). Then the expectation value of $O$ vanishes on physical states $|\psi\rangle$ from $\langle\psi|O| \psi\rangle=$ $\langle\psi|[H, A]| \psi\rangle=0$. The mistake in this argument is easily detected by replacing $H$ with a nonrelativistic free particle quantum hamiltonian, $A$ with $x$, and $|\psi\rangle$ with an eigenstate of the momentum: The error is to neglect the infinities generated by the use of generalized states.
}

Living Reviews in Relativity (1998-1)

http://www.livingreviews.org 
to the solution of the problem of extracting the classical limit (of the dynamics) from the theory.

Matter. The basics of the description of matter in the loop formalism have been

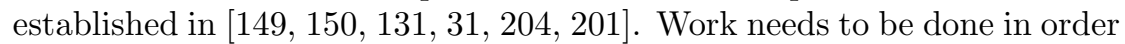
to develop a full description of the basic matter couplings. In particular, there are strong recurring indications that the Planck scale discreteness naturally cuts the traditional quantum field infinities off. In particular, in [ZII]], Thiemann argues that the Hamiltonian constraint governing the coordinate time evolution of the Yang-Mills field is a well defined operator (I recall that, due to the ultraviolet divergences, no rigorously well defined hamiltonian operator for conventional Yang-Mills theory is known in 4 dimensions.) If these indications are confirmed, the result would be very remarkable. What is still missing are calculational techniques that could allow us to connect the well-defined constraint with finite observables quantities such as scattering amplitudes.

Spacetime formalism. In my view, the development of continuous spacetime formalisms, [181, 160, [26, 143], is one of the most promising areas of development of the theory, because it might be the key for addressing most of the open problems. First, a spacetime formalism frees us from the obscurities of the frozen time formalism, and allows an intuitive, Feynman-style, description of the dynamics of quantum spacetime. I think that the classical limit, the quantum description of black holes, or graviton-graviton scattering, just to mention a few examples, could be addressed much more easily in the covariant picture. Second, it allows the general ideas of Hartle [102] and Isham [118, ए1. ए22, ए21] on the interpretation of generally covariant quantum theories to be applied in loop quantum gravity. This could drastically simplify the complications of the canonical way of dealing with general covariant observables [169, [67]. Third, the spacetime formalism should suggest solutions to the problem of selecting the correct hamiltonian constraint: it is usually easier to deal with invariances in the Lagrangian rather than in the hamiltonian formalisms. The spacetime formalism is just born, and much has to be done. See the original papers for suggestions and open problems.

Black holes. The derivation of the Bekenstein-Hawking entropy formula is a major success of loop quantum gravity, but much remains to be understood. A clean derivation from the full quantum theory is not yet available. Such a derivation would require us to understand what, precisely, the event horizon in the quantum theory is. In other words, given a quantum state of the geometry, we should be able to define and "locate" its horizon (or whatever structure replaces it in the quantum theory). To do so, we should understand how to effectively deal with the quantum dynamics, how to describe the classical limit (in order to find the quantum states corresponding to classical black hole solutions), as well as how to describe asymptotically flat quantum states.

Living Reviews in Relativity (1998-1) http://www.livingreviews.org 
Besides these formal issues, at the roots of the black hole entropy puzzle there is a basic physical problem, which, to my understanding, is still open. The problem is to understand how we can use basic thermodynamical and statistical ideas and techniques in a general covariant context. [0 To appreciate the difficulty, notice that statistical mechanics makes heavy use of the notion of energy (say in the definition of the canonical or microcanonical ensembles); but there is no natural local notion of energy associated to a black hole (or there are too many of such notions). Energy is an extremely slippery notion in gravity. Thus, how do we define the statistical ensemble? Put in other words: To compute the entropy (say in the microcanonical) of a normal system, we count the states with a given energy. In GR we should count the states with a given what? One may say: black hole states with a given area. But why so? We do understand why the number of states with given energy governs the thermodynamical behavior of normal systems. But why should the number of states with given area govern the thermodynamical behavior of the system, namely govern its heat exchanges with the exterior? A tentative physical discussion of this last point can be found in [ [78] .

How to extract physics from the theory. Assume we pick a specific hamiltonian constraint. Then we have, in principle, a well defined quantum theory. How do we extract physical information from it? Some physical consequences of the theory, such as the area and volume eigenvalues, or the entropy formula, have been extracted from the theory by various ad hoc methods. But is there a general technique, say corresponding to the traditional QFT perturbation expansion of the $S$ matrix, for describing the full dynamics of the gravitational field? Presumably, such general techniques should involve some kind of expansion, since we could not hope to solve the theory exactly. Attempts to define physical expansions have been initiated in [1755] and, in different form, in [160]]. Ideally, one would want a general scheme for computing transition amplitudes in some expansion parameter around some state. Computing scattering amplitudes would be of particular interest, in order to make connection with particle physics language and to compare the theory with string predictions.

Classical limit. Finally, to prove that loop quantum gravity is a valuable candidate for describing quantum spacetime, we need to prove that its classical limit is GR (or at least overlaps GR in the regime where GR is well tested). The traditional connection between loop quantum gravity and classical GR is via the notion of weave, a quantum state that "looks semiclassical" at distances large compared to the Planck scale. However, the weaves studied so far [24, 9.9] are 3d weaves, in the sense that they are eigenstates of the three dimensional metric. Such a state corresponds to an eigenstate of the position for a particle. Classical behavior is recovered

\footnotetext{
${ }^{10} \mathrm{~A}$ general approach to this problem, and an idea about its connection with the "problem of time" in quantum gravity, have been developed by Connes and Rovelli in [ [773, ए74, 69].
}

Living Reviews in Relativity (1998-1)

http://www.livingreviews.org 
not by these states but rather by wave packets which have small spread in position as well as in momentum. Similarly, the quantum Minkowski spacetime should have small spread in the three metric as well as in its momentum - as the quantum electromagnetic vacuum has small quantum spread in the electric and magnetic field. To recover classical GR from loop quantum gravity, we must understand such states. Preliminary investigation in this direction can be found in [12.5, 1124], but these papers are now several years old, and they were written before the more recent solidification of the basics of the theory. Another direction consists in the direct study of coherent states in the state space of the theory.

As these brief notes indicate, the various open problems in loop quantum gravity are interconnected. In a sense, loop quantum gravity grew aiming at the nonperturbative regime, and the physical results obtained so far are in this regime. The main issue is then to recover the long distance behavior of the theory. That is, to study its classical limit and the dynamics of the low energy excitations over a semiclassical background. Understanding this aspect of the theory would assure us that the theory we are dealing with is indeed a quantum theory of the gravitational field, would allow us to understand quantum black holes, would clarify the origin of infinities in the matter hamiltonians and so on. Still, in other words, what mostly needs to be understood is the structure of the (Minkowski) vacuum in loop quantum gravity.

Living Reviews in Relativity (1998-1) http://www.livingreviews.org 


\section{Short Summary and Conclusion}

In this section, I very briefly summarize the state of loop quantum gravity and its main results. The mathematics of the theory is solidly defined, and is understood from several alternative points of view. Long standing problems such as the lack of a scalar product, the difficult of controlling the overcompleteness of the loop basis and the problem of implementing the reality condition in the quantum theory have been successfully solved or sidestepped. The kinematics is given by the Hilbert space $\mathcal{H}$, defined in Section [6.2, which carries a representation of the basic operators: the loop operators $(22-23)$. A convenient orthonormal basis in $\mathcal{H}$ is provided by the spin network states, defined in Section 6.3. The diffeomorphism invariant states are given by the s-knot states, and the structure and properties of the (diff-invariant) quantum states of the geometry are quite well understood (Section 6.8). These states give a description of quantum spacetime in terms of polymer-like excitations of the geometry. More precisely, in terms of elementary excitations carrying discretized quanta of area.

The dynamics is coded into the hamiltonian constraint. A well defined version of this constraint exists (see equation (35)), and thus a consistent theory exists, but a proof that the classical limit of this theory is classical general relativity is still lacking. Alternative versions of the hamiltonian constraint have been proposed and are under investigation. In all these cases, the hamiltonian has the crucial properties of acting on nodes only. This implies that its action is naturally discrete and combinatorial. This fact is possibly at the roots of the finiteness of the theory. A large class of physical states which are exact solutions of the dynamics are given by s-knots without nodes; other exact states are related to knot theory invariants (Section 7.1).

The theory can be extended to include matter, and there are strong indications that ultraviolet divergences do not appear. A spacetime covariant version of the theory, in the form of a topological sum over surfaces, is under development (Section 6.10).

The main physical results derived so far from the theory are given by the explicit computation of the eigenvalues of area and volume, some of which are given in equations (41-45), and a derivation of the black hole entropy formula (Section 41). The two main (related) open problems are to understand the description of the low energy regime within the theory and to choose the correct version of the hamiltonian constraint.

\subsection{Conclusion}

The history of quantum gravity is a sequel of moments of great excitement followed by bitter disappointments. I distinctively remember, as a young student, listening to a very famous physicist announcing at a major conference that quantum gravity was solved (I think it was the turn of supergravity). The list of theories that claimed to be final and have then ended up forgotten or superseded is a reason of embarrassment for the theoretical physics community,

Living Reviews in Relativity (1998-1)

http://www.livingreviews.org 
in my opinion.

In my view, loop quantum gravity is the best we can do so far in trying to understand quantum spacetime, from a nonperturbative, background-independent point of view. Theoretically, we have reasons to suspect that this approach could represent a consistent quantum theory with the correct classical limit, but there are also some worrying contrary indications. The theory yields a definite physical picture of quantum spacetime and definite quantitative predictions, but a systematic way of extracting physical information is still lacking. Experimentally, there is no support to the theory, neither direct nor indirect. The spectra of area and volume computed in the theory could or could not be physically correct. I hope I live long enough to know! 


\section{Acknowledgments}

I am especially indebted to Michael Reisenberger, Roberto DePietri Don Marolf, Jerzy Lewandowski, John Baez, Thomas Thiemann and Abhay Ashtekar for their accurate reading of the manuscript, their detailed suggestions and their ferocious criticisms. Their inputs have been extended and their help precious. I am grateful to all my friends in the community for the joy of doing physics together. This work was supported by NSF Grant PHY-95-15506.

Living Reviews in Relativity (1998-1)

http: //www . livingreviews .org 


\section{References}

[1] Agishtein, M., and Migdal, A., "Critical behavior of dynamically triangulated quantum gravity in 4 dimensions", Nucl. Phys., 385, 395-412, (1992). For a related online version see: M. Agishtein, et al., "Critical behavior of dynamically triangulated quantum gravity in 4 dimensions", (April, 1992), [Online Los Alamos Preprint Archive]: cited on 29 September 1997, http://xxx.lanl.gov/abs/hep-lat/9204004. 2

[2] Albert Einstein Institute, "Max Planck Institute for Gravitational Physics", (1997), [Online HTML Document]: cited on 29 September 1997, http://www.aei-potsdam.mpg.de/. [

[3] Amati, D., Ciafaloni, M., and Veneziano, G., "Superstring collisions at Planckian energies", Phys. Lett. B, 197, 81-88, (1987). 2.3

[4] Amati, D., Ciafaloni, M., and Veneziano, G., "Classical and quantum gravity effects from Planckian energy superstring collisions", Int. J. Mod. Phys., 3, 1615-1661, (1988). 2.3

[5] Amati, D., Ciafaloni, M., and Veneziano, G., "Can spacetime be probed below the string size?", Phys. Lett. B, 216, 41-47, (1989). 2.3

[6] Amati, D., Ciafaloni, M., and Veneziano, G., "Planckian scattering beyond the semiclassical approximation", Phys. Lett. B, 289, 87-91, (1992). [2.3]

[7] Ambjorn, J., Carfora, M., and Marzuoli, A., "The geometry of dynamical triangulations", (December, 1996), [Online Los Alamos Preprint Archive]: cited on 29 September 1997, http://xxx.lanl.gov/abs/ hep-th/9612069. 2

[8] Ashtekar, A., "New variables for classical and quantum gravity", Phys. Rev. Lett., 57(18), 2244-2247, (1986). 3, 6

[9] Ashtekar, A., "New Hamiltonian formulation of general relativity", Phys. Rev. D, 36(6), 1587-1602, (1987). 3

[10] Ashtekar, A., Lecture notes on non-perturbative canonical gravity. Notes prepared in collaboration with $R$ Tate. Advanced Series in Astrophysics and Cosmology, Volume 6, (World Scientific, Singapore, 1991). 迁, 6, 6

[11] Ashtekar, A., "Mathematical problems of non-perturbative quantum general relativity", in Julia, B., ed., Gravitation and Quantization, Les Houches, Session LVIII, 1992, (Elsevier, Amsterdam, 1995). 田

[12] Ashtekar, A., Baez, J.C., Corichi, A., and Krasnov, K., private communication. [3, 6, 7.2

Living Reviews in Relativity (1998-1)

http://www.livingreviews.org 
[13] Ashtekar, A., and C.J., Isham, "Representations of the holonomy algebras of gravity and non-abelian gauge theories", Class. Quantum Grav., 9, 1433-1485, (1992). 3, 6.1, 6.6, 6.7

[14] Ashtekar, A., Husain, V., Rovelli, C., Samuel, J., and Smolin, L., "2 +1 quantum gravity as a toy model for the $3+1$ theory", Class. Quantum Grav., 6, L185-L193, (1989). 3, 7.1

[15] Ashtekar, A., and Lewandowski, J., "Representation theory of analytic holonomy $C^{\star}$ algebras", in Baez, J.C., ed., Knots and quantum gravity, 21-61, (Oxford University Press, Oxford, 1994). 63, 6.2, 6.2, 6.6, 6.7, 8

[16] Ashtekar, A., and Lewandowski, J., "Differential geometry on the space of connections via graphs and projective limits", J. Geom. Phys., 17, 191230, (1995). For a related online version see: A. Ashtekar, et al., "Differential geometry on the space of connections via graphs and projective limits", (December, 1994), [Online Los Alamos Preprint Archive]: cited on 29 September 1997, http://xxx.lanl.gov/abs/hep-th/9412073. [3, [.], 6.2, [6.2, 6.6, 6.7, 8

[17] Ashtekar, A., and Lewandowski, J., "Projective techniques and functional integration for gauge theories", J. Math. Phys., 36, 2170-2191, (1995). For a related online version see: A. Ashtekar, et al., "Projective techniques and functional integration", (November, 1994), [Online Los Alamos Preprint Archive]: cited on 29 September 1997, http: //xxx.lanl.gov/abs/hep-th/9411046. 3, 6.2, 6.2, 6.6, 6.7, 8

[18] Ashtekar, A., and Lewandowski, J., "Quantum Theory of Gravity I: Area Operators", Class. Quantum Grav., 14, A55-A81, (1997). For a related online version see: A. Ashtekar, et al., "Quantum Theory of Gravity I: Area Operators", (February, 1996), [Online Los Alamos Preprint Archive]: cited on 29 September 1997, http://xxx.lanl.gov/abs/ gr-qc/9602046. 33, 6.1, 6.3, 6.5, 7.2, 7.2, 7.2, 7.2

[19] Ashtekar, A., Lewandowski, J., Marolf, D., Mourão, J., and Thiemann, T., "Quantization of diffeomorphism invariant theories of connections with local degrees of freedom", J. Math. Phys., 36, 6456-6493, (1995). For a related online version see: A. Ashtekar, et al., "Quantization of diffeomorphism invariant theories of connections with local degrees of freedom", (April, 1995), [Online Los Alamos Preprint Archive]: cited on 29 September 1997, http://xxx.lanl.gov/abs/gr-qc/9504018. 3, 田, 6.5, 6.7, 6.8

[20] Ashtekar, A., Lewandowski, J., Marolf, D., Mourao, J., and Thiemann, T., "SU $N)$ Quantum Yang-Mills theory in two dimensions: A complete solution", J. Math. Phys., 38(11), 5453-5482, (1997). For a related online version see: A. Ashtekar, et al., "Closed formula for Wilson loops for $S U(N)$ Quantum Yang-Mills Theory in two dimensions", (May, 1996),

Living Reviews in Relativity (1998-1)

http://www.livingreviews.org 
[Online Los Alamos Preprint Archive]: cited on 29 September 1997, http: //xxx.lanl.gov/abs/hep-th/9605128. To appear. 3, 5

[21] Ashtekar, A., and Loll, R., "New Loop Representation for 2+1 Gravity", Class. Quantum Grav., 11, 2417-2434, (1994). For a related online version see: A. Ashtekar, et al., "New Loop Representation for 2+1 Gravity", (May, 1994), [Online Los Alamos Preprint Archive]: cited on 29 September 1997, http://xxx.lanl.gov/abs/gr-qc/9405031. 3, 7.1

[22] Ashtekar, A., and Rovelli, C., "A loop representation for the quantum Maxwell field", Class. Quantum Grav., 9, 1121-1150, (1992). 3, 7.1

[23] Ashtekar, A., Rovelli, C., and Smolin, L., "Gravitons and loops", Phys. Rev. D, 44(6), 1740-1755, (1991). 3

[24] Ashtekar, A., Rovelli, C., and Smolin, L., "Weaving a classical geometry with quantum threads", Phys. Rev. Lett., 69, 237-240, (1992). 3, 6.5, [.2, [.2.2, ए0

[25] Baez, J.C., "Strings, loops, knots and gauge fields", (September, 1993), [Online Los Alamos Preprint Archive]: cited on 29 September 1997, http: //xxx.lanl.gov/abs/hep-th/9309067. [3]

[26] Baez, J.C., "Spin foam models", (September, 1997), [Online Los Alamos Preprint Archive]: cited on 29 September 1997, http://xxx.lanl.gov/ abs/gr-qc/9709052. 3, 6.10, 8, 9

[27] Baez, J.C., "This week's finds in mathematical physics", (September, 1997), [Online HTML Document]: cited on 29 September 1997, http: //math.ucr.edu/home/baez/README.html\\#TWF. \$

[28] Baez, J.C., "Diffeomorphism-invariant generalized measures on the space of connections modulo gauge transformations", in Crane, L., and Yetter, D., eds., Proceedings of the Conference on Quantum Topology, 213223, (World Scientific, Singapore, 1994). For a related online version see: J.C. Baez, "Diffeomorphism-invariant generalized measures on the space of connections modulo gauge transformations", (May, 1995), [Online Los Alamos Preprint Archive]: cited on 29 September 1997, http: //xxx.lanl.gov/abs/hep-th/9305045. 3, 3]

[29] Baez, J.C., "Generalized Measures in Gauge Theory", Lett. Math. Phys., 31, 213-223, (1994). For a related online version see: J.C. Baez, "Diffeomorphism invariant generalized measures on the space of connections modulo gauge transformations", (May, 1993), [Online Los Alamos Preprint Archive]: cited on 29 September 1997, http://xxx.lanl.gov/abs/ hep-th/9305045. [3]

[30] Baez, J.C., "Generalized Measures in Gauge Theory", Lett. Math. Phys., 31, 213-224, (1994). For a related online version see: J.C. Baez, "Generalized Measures in Gauge Theory", (October, 1993), [Online Los Alamos

Living Reviews in Relativity (1998-1) http://www.livingreviews.org 
Preprint Archive]: cited on 29 September 1997, http://xxx.lanl.gov/ abs/hep-th/9310201. B

[31] Baez, J.C., "Generalized Measures in Gauge Theory", Lett. Math. Phys., 31, 213-223, (1994). 3, 6.7, 9

[32] Baez, J.C., Knots and Quantum Gravity, (Oxford University Press, Oxford, 1994). [1

[33] Baez, J.C., "Strings, Loops, Knots and Gauge Fields", in Baez, J.C., ed., Knots and Quantum Gravity, 133-159, (Cambridge University Press, Cambridge, 1994). For a related online version see: J.C. Baez, "Strings, Loops, Knots and Gauge Fields", (September, 1993), [Online Los Alamos Preprint Archive]: cited on 29 September 1997, http://xxx.lanl.gov/ abs/hep-th/9309067. 6.10

[34] Baez, J.C., "Spin Networks in Gauge Theory", Adv. Math., 117(2), 253272, (1996). For a related online version see: J.C. Baez, "Spin Network States in Gauge Theory", (November, 1994), [Online Los Alamos Preprint Archive]: cited on 29 September 1997, http://xxx.lanl.gov/ abs/gr-qc/9411007. [3, 3, 6.3

[35] Baez, J.C., "Spin Networks in Nonperturbative Quantum Gravity", in Kauffman, L.H., ed., The Interface of Knots and Physics, (American Mathematical Society, Providence, Rhode Island, 1996). For a related online version see: J.C. Baez, "Spin Networks in Nonperturbative Quantum Gravity", (April, 1995), [Online Los Alamos Preprint Archive]: cited on 29 September 1997, http://xxx.lanl.gov/abs/gr-qc/9504036. 3, 6.3

[36] Baez, J.C., and Krasnov, K., "Quantization of diffeomorphism invariant theories with fermions", (March, 1997), [Online Los Alamos Preprint Archive]: cited on 29 September 1997, http://xxx.lanl.gov/abs/ hep-th/9703112. [3, 7.1]

[37] Baez, J.C., and Muniain, J., Gauge Fields, Knots, and Gravity, (World Scientific Press, Singapore, 1994). 4

[38] Balachandran, A.P., Chandar, L., and Momen, A., "Edge states in Gravity and Black Hole Physics", Nucl. Phys. B, 461, 581-596, (1996). For a related online version see: A.P. Balachandran, et al., "Edge states in Gravity and Black Hole Physics", (December, 1994), [Online Los Alamos Preprint Archive]: cited on 29 September 1997, http://xxx.lanl.gov/ abs/gr-qc/9412019. 7.2

[39] Balachandran, A.P., Chandar, L., and Momen, A., "Edge States and Entanglement Entropy", Int. J. Mod. Phys. A, 12(3), 625-641, (1997). For a related online version see: A.P. Balachandran, et al., "Edge States and Entanglement Entropy", (December, 1995), [Online Los Alamos Preprint Archive]: cited on 29 September 1997, http://xxx.lanl.gov/abs/ hep-th/9512047. 7.2

Living Reviews in Relativity (1998-1)

http://www.livingreviews.org 
[40] Barbero, F., "Real-polynomial formulation of general relativity in terms of connections", Phys. Rev. D, 49, 6935-6938, (1994). 33, 3

[41] Barbero, F., "Real Ashtekar Variables for Lorentzian Signature Spacetimes", Phys. Rev. D, 51, 5507-5510, (1995). For a related online version see: F. Barbero, "Real Ashtekar Variables for Lorentzian Signature Spacetimes", (October, 1994), [Online Los Alamos Preprint Archive]: cited on 29 September 1997, http://xxx.lanl.gov/abs/gr-qc/9410014. 3, 33, 6

[42] Barbero, F., "Reality Conditions and Ashtekar Variables: a Different Perspective", Phys. Rev. D, 51, 5498-5506, (1995). For a related online version see: F. Barbero, "Reality Conditions and Ashtekar Variables: a Different Perspective", (October, 1994), [Online Los Alamos Preprint Archive]: cited on 29 September 1997, http://xxx.lanl.gov/ abs/gr-qc/9410013. 3, [3

[43] Barbero, F., "From Euclidean to Lorentzian General Relativity: The Real Way", Phys. Rev. D, 54, 1492-1499, (1996). For a related online version see: F. Barbero, "From Euclidean to Lorentzian General Relativity: The Real Way", (May, 1996), [Online Los Alamos Preprint Archive]: cited on 29 September 1997, http://xxx.lanl.gov/abs/gr-qc/9605066. 3, 3]

[44] Barreira, M., Carfora, M., and Rovelli, C., "Physics with nonperturbative quantum gravity: radiation from a quantum black hole", Gen. Relativ. Gravit., 28, 1293-1299, (1996). For a related online version see: M. Barreira, et al., "Physics with nonperturbative quantum gravity: radiation from a quantum black hole", (March, 1996), [Online Los Alamos Preprint Archive]: cited on 29 September 1997, http://xxx.lanl.gov/ abs/gr-qc/9603064. 7.2

[45] Beetle, C., and Corichi, A., "Bibliography of Publications related to Class. and Quantum Grav. in terms of Connection and Loop Variables", (March, 1997), [Online Los Alamos Preprint Archive]: cited on 29 September 1997, http://xxx.lanl.gov/abs/gr-qc/9703044. 专

[46] Bekenstein, J.D., "Black holes and entropy", Phys. Rev. D, 7, 2333-2346, (1973). 7.2

[47] Bekenstein, J.D., and Mukhanov, V.F., "Spectroscopy of the quantum black hole", (May, 1995), [Online Los Alamos Preprint Archive]: cited on 29 September 1997, http://xxx.lanl.gov/abs/gr-qc/9505012. 7.2

[48] Blencowe, M.P., "The Hamiltonian constraint in quantum gravity", Nulc. Phys. B, 341(1), 213-251, (1990). 迆

[49] Borissov, R., "Graphical Evolution of Spin Netwoks States", (June, 1996), [Online Los Alamos Preprint Archive]: cited on 29 September 1997, http: 7/xxx.lanl.gov/abs/gr-qc/9606013. [3, 7.1] 
[50] Borissov, R., "Weave states for plane gravitational waves", Phys. Rev. D, 49, 923-929, (1994). 7.2

[51] Borissov, R., "Regularization of the Hamiltonian constraint and the closure of the constraint algebra", Phys. Rev. D, 55, 2059-2068, (1997). For a related online version see: R. Borissov, "Regularization of the Hamiltonian constraint and the closure of the constraint algebra", (November, 1994), [Online Los Alamos Preprint Archive]: cited on 29 September 1997, http://xxx.lanl.gov/abs/gr-qc/9411038. 3

[52] Borissov, R., DePietri, R., and Rovelli, C., "Matrix elements of Thiemann hamiltonian", Class. Quantum Grav., To appear, (1997). For a related online version see: R. Borissov, et al., "Matrix elements of Thiemann hamiltonian", (March, 1997), [Online Los Alamos Preprint Archive]: cited on 29 September 1997, http://xxx.lanl.gov/abs/gr-qc/9703090. 6.3, [..9, 7.2. $7.2,7.2$

[53] Brink, D.M., and Satchler, R., Angular Momentum, (Claredon Press, Oxford, 1968). 6.4

[54] Brügmann, B., Personal communication. 8

[55] Brügmann, B., On the constraints of quantum general relativity in the loop representation, PhD Thesis, (Syracuse University, Syracuse, New York, 1991). 3

[56] Brügmann, B., "Loop representation", in Ehlers, J., and Friedrich, H., eds., Canonical Gravity: From Classical to Quantum, (Springer-Verlag, Berlin, 1993). 7.1

[57] Brügmann, B., Gambini, R., and Pullin, J., "Jones polynomials for intersecting knots as physical states of quantum gravity", Nulc. Phys. B, 385, 587-603, (1992). 3, 7.1

[58] Brügmann, B., Gambini, R., and Pullin, J., "Knot invariants as nondegenerate quantum geometries", Phys. Rev. Lett., 68(4), 431-434, (1992). 3, 7.1

[59] Brügmann, B., Gambini, R., and Pullin, J., "How the Jones polynomial gives rise to physical states of quantum general relativity", Gen. Relativ. Gravit., 25, 1-6, (1993). 3, 7.1

[60] Brügmann, B., and Pullin, J., "Intersecting N loop solutions of the Hamiltonian constraint of Quantum Gravity", Nulc. Phys. B, 363, 221-244, (1991). B

[61] Brügmann, B., and Pullin, J., "On the constraints of quantum gravity in the loop representation", Nulc. Phys. B, 390, 399-438, (1993). 3

Living Reviews in Relativity (1998-1)

http://www.livingreviews.org 
[62] Brügmann, B., B., and Marinari, E., "4d Simplicial Quantum Gravity with a Non-Trivial Measure", Phys. Rev. Lett., 70, 1908-1911, (1993). For a related online version see: B. Brügmann, B., et al., "4d Simplicial Quantum Gravity with a Non-Trivial Measure", (October, 1992), [Online Los Alamos Preprint Archive]: cited on 29 September 1997, http://xxx. lanl.gov/abs/hep-lat/9210002. 2

[63] Carlip, S., "Observables, gauge invariance and time in $2+1$ dimensional gravity", Phys. Rev. D, 42, 2647-2654, (1990). 7.1

[64] Carlip, S., "Statistical Mechanics and Black Hole Thermodynamics", Nucl. Phys. B, Proc. Suppl. 57, 8-12, (1997). For a related online version see: S. Carlip, "Statistical Mechanics and Black Hole Thermodynamics", (February, 1997), [Online Los Alamos Preprint Archive]: cited on 29 September 1997, http://xxx.lanl.gov/abs/gr-qc/9702017. 7.2

[65] Carlip, S., "Statistical Mechanics of the Three-Dimensional Euclidean Black Hole", Phys. Rev. D, 55(2), 878-882, (1997). For a related online version see: S. Carlip, "The Statistical Mechanics of the ThreeDimensional Euclidean Black Hole", (June, 1996), [Online Los Alamos Preprint Archive]: cited on 29 September 1997, http://xxx.lanl.gov/ abs/gr-qc/9606043. 7.2

[66] Catterall, S., Kogut, J., and Renken, R., "Phase Structure of Four Dimensional Simplicial Quantum Gravity", Phys. Lett. B, 328, 277-283, (1994). For a related online version see: S. Catterall, et al., "Phase Structure of Four Dimensional Simplicial Quantum Gravity", (October, 1994), [Online Los Alamos Preprint Archive]: cited on 29 September 1997, http://xxx.lanl.gov/abs/hep-lat/9401026. 2

[67] Citanovic̀, P., Group theory, (Nordita classical illustrated, Copenhagen, 1984). 6.4

[68] Connes, A., Non Commutative Geometry, (Academic Press, S. Diego, 1994). 2

[69] Connes, A., and Rovelli, C., "Von Neumann algebra automorphisms and time versus thermodynamics relation in general covariant quantum theories", Class. Quantum Grav., 11(12), 2899-2917, (1994). For a related online version see: A. Connes, et al., "Von Neumann algebra automorphisms and time versus thermodynamics relation in general covariant quantum theories", (June, 1994), [Online Los Alamos Preprint Archive]: cited on 29 September 1997, http://xxx.lanl.gov/abs/gr-qc/9406019. 10

[70] Corichi, A., and Krasnov, K., "Loop Quantization of Maxwell Theory and Electric Charge Quantization", (March, 1997), [Online Los Alamos Preprint Archive]: cited on 29 September 1997, http://xxx.lanl.gov/ abs/hep-th/9703177. 6, 7.2 
[71] Crane, L., "Topological field theory as the key to quantum gravity", in Baez, J.C., ed., Knots and Quantum Gravity, 121-131, (Oxford University Press, Oxford, 1994). 6.10

[72] Crane, L., "Clock and Categories: Is Quantum Gravity Algebraic?", J. Math. Phys., 36, 6180-6193, (1995). 6.10

[73] Crane, L., and Frenkel, I., "Four-dimensional topological field theory, Hopf categories and the canonical bases", J. Math. Phys., 35(10), 5136-5154, (1994). For a related online version see: L. Crane, et al., "Four-dimensional topological field theory, Hopf categories and the canonical bases", (May, 1994), [Online Los Alamos Preprint Archive]: cited on 29 September 1997, http://xxx.lanl.gov/abs/hep-th/9405183. 6.10

[74] Crane, L., Kauffman, L.H., and Yetter, D.N., "Evaluating the CraneYetter Invariant", (September, 1993), [Online Los Alamos Preprint Archive]: cited on 29 September 1997, http://xxx.lanl.gov/abs/ hep-th/9309063. 6.10

[75] Crane, L., and Yetter, D., "A Categorical Construction of 4d TQFTs", in Baadhio, R., and Kauffman, L.H., eds., Quantum Topology, (World Scientific, Singapore, 1993). For a related online version see: L. Crane, et al., "A Categorical Construction of 4d TQFTs", (January, 1993), [Online Los Alamos Preprint Archive]: cited on 29 September 1997, http://xxx. lanl.gov/abs/hep-th/9301062. 6.10

[76] DePietri, R., "On the relation between the connection and the loop representation of quantum gravity", Class. Quantum Grav., 14, 53-69, (1997). For a related online version see: R. DePietri, "On the relation between the connection and the loop representation of quantum gravity", (May, 1996), [Online Los Alamos Preprint Archive]: cited on 29 September 1997, http://xxx.lanl.gov/abs/gr-qc/9605064. 3, 6.6

[77] DePietri, R., and Rovelli, C., "Geometry Eigenvalues and Scalar Product from Recoupling Theory in Loop Quantum Gravity", Phys. Rev. D, 54, 2664-2690, (1996). For a related online version see: R. DePietri, et al., "Geometry Eigenvalues and Scalar Product from Recoupling Theory in Loop Quantum Gravity", (February, 1996), [Online Los Alamos Preprint Archive]: cited on 29 September 1997, http://xxx.lanl.gov/ abs/gr-qc/9602023. 63, 田, 6.1, 6.3, 6.4, 6.4, 6.5, 6.7, 6.7, 6.9, 7.2, 7.2, प्य

[78] Di Bartolo, C., Gambini, R., and Griego, "Extended loop representation of quantum gravity", Phys. Rev. D, 51(2), 502-516, (1995). For a related online version see: C. Di Bartolo, et al., "Extended loop representation of quantum gravity", (June, 1994), [Online Los Alamos Preprint Archive]: cited on 29 September 1997, http://xxx.lanl.gov/abs/ gr-qc/9406039. 3, 国

Living Reviews in Relativity (1998-1)

http://www.livingreviews.org 
[79] Di Bartolo, C., Gambini, R., and Griego, "Lattice knot theory and quantum gravity in the loop representation", Phys. Rev. D, 56, 2127-2143, (1997). For a related online version see: C. Di Bartolo, et al., "Lattice knot theory and quantum gravity in the loop representation", (August, 1996), [Online Los Alamos Preprint Archive]: cited on 29 September 1997, http://xxx.lanl.gov/abs/gr-qc/9608033. 3, 7.1

[80] Di Bartolo, C., Gambini, R., Griego, J., and Pullin, J., "Extended loops: A new arena for nonperturbative quantum gravity", Phys. Rev. Lett., 72, 3638-3641, (1994). 3, 国

[81] Ehlers, J., and Friedrich, H., eds., Canonical Gravity: from Classical to Quantum, (Springer-Verlag, Berlin, 1994). 6

[82] Ezawa, K., "Nonperturbative solutions for canonical quantum gravity: an overview", (October, 1996), [Online Los Alamos Preprint Archive]: cited on 29 September 1997, http://xxx.lanl.gov/abs/gr-qc/9601050. 7.1]

[83] Foxon, T.J., "Spin networks, Turaev - Viro theory and the loop representation", Class. Quantum Grav., 12(4), 951-964, (1995). 6.10

[84] Fritelli, S., Lehner, L., and Rovelli, C., "The complete spectrum of the area from recoupling theory in loop quantum gravity", Class. Quantum Grav., 13, 2921-2932, (1996). For a related online version see: S. Fritelli, et al., "The complete spectrum of the area from recoupling theory in loop quantum gravity", (August, 1996), [Online Los Alamos Preprint Archive]: cited on 29 September 1997, http://xxx.lanl.gov/abs/gr-qc/9608043. [3, [7.2, $7.2,7.2$

[85] Frittelli, S., Kozameh, N.C., and Newman, "GR via Characteristic Surfaces", J. Math. Phys., 36, 4984-5004, (1995). For a related online version see: S. Frittelli, et al., "GR via Characteristic Surfaces", (February, 1995), [Online Los Alamos Preprint Archive]: cited on 29 September 1997, http://xxx.lanl.gov/abs/gr-qc/9502028. 2

[86] Frittelli, S., Kozameh, N.C., Newman, E.T., Rovelli, C., and Tate, R.T., "Fuzzy spacetime from a null-surface version of GR", Class. Quantum Grav., 14, A143-A154, (1997). For a related online version see: S. Frittelli, et al., "Fuzzy spacetime from a null-surface version of GR", (March, 1996), [Online Los Alamos Preprint Archive]: cited on 29 September 1997, http: //xxx.lanl.gov/abs/gr-qc/9603061. 2

[87] Frittelli, S., Kozameh, N.C., Newman, E.T., Rovelli, C., and Tate, R.T., "On the quantization of the Null-Surface formulation of GR", Phys. Rev. $D, \mathbf{5 6}, 889-907,(1997)$. For a related online version see: S. Frittelli, et al., "On the quantization of the Null-Surface formulation of GR", (December, 1996), [Online Los Alamos Preprint Archive]: cited on 29 September 1997, http://xxx.lanl.gov/abs/gr-qc/9612010. 2] 
[88] Gambini, R., Garat, A., and Pullin, J., "The constraint algebra of quantum gravity in the loop representation", Int. J. Mod. Phys. D, 4(5), 589616, (1995). For a related online version see: R. Gambini, et al., "The constraint algebra of quantum gravity in the loop representation", (April, 1994), [Online Los Alamos Preprint Archive]: cited on 29 September 1997, http://xxx.lanl.gov/abs/gr-qc/9404059. 3

[89] Gambini, R., Griego, J., and Pullin, J., "Chern-Simons states in spinnetwork quantum gravity", (March, 1997), [Online Los Alamos Preprint Archive]: cited on 29 September 1997, http://xxx.lanl.gov/abs/ gr-qc/9703042. 3

[90] Gambini, R., Lewandowski, J., Marolf, D., and Pullin, J., "On the consistency of the constraint algebra in spin network quantum gravity", (September, 1997), [Online Los Alamos Preprint Archive]: cited on 29 September 1997, http://xxx.lanl.gov/abs/? [3, 8

[91] Gambini, R., and Pullin, J., "Quantum Einstein-Maxwell fields: a unified viewpoint from the loop representation", Phys. Rev. D, 47, R5214-R5218, (1993). For a related online version see: R. Gambini, et al., "Quantum Einstein-Maxwell fields: a unified viewpoint from the loop representation", (October, 1992), [Online Los Alamos Preprint Archive]: cited on 29 September 1997, http://xxx.lanl.gov/abs/hep-th/9210110. 7.1

[92] Gambini, R., and Pullin, J., "The Gauss linking number in quantum gravity", in Baez, J.C., ed., Knots and quantum gravity, 63-76, (Oxford University Press, Oxford, 1994). 3

[93] Gambini, R., and Pullin, J., Loops, Knots, Gauge Theory and Quantum Gravity, (Cambridge University Press, Cambridge, 1996). 团

[94] Gambini, R., and Pullin, J., "A rigorous solution of the quantum Einstein equations", Phys. Rev. D, 54, 5935-5938, (1996). For a related online version see: R. Gambini, et al., "A rigorous solution of the quantum Einstein equations", (November, 1995), [Online Los Alamos Preprint Archive]: cited on 29 September 1997, http://xxx.lanl.gov/abs/ gr-qc/9511042. [3, 7.1

[95] Gambini, R., and Trias, A., "On the geometrical origin of gauge theories", Phys. Rev. D, 23, 553-555, (1981). 3, 6.1

[96] Gambini, R., and Trias, A., "Gauge dynamics in the C representation", Nucl. Phys. B, 278, 436-448, (1986). 3, 6.1

[97] Garay, L.J., "Quantum gravity and minimum length", (march, 1994), [Online Los Alamos Preprint Archive]: cited on 29 September 1997, http: //xxx.lanl.gov/abs/gr-qc/9403008. 7.2 
[98] Grot, N., and Rovelli, C., "Moduli-space of knots with intersections", J. Math. Phys., 37, 3014-3021, (1996). For a related online version see: N. Grot, et al., "Moduli-space of knots with intersections", (April, 1996), [Online Los Alamos Preprint Archive]: cited on 29 September 1997, http: 7/xxx.lanl.gov/abs/gr-qc/9604010. 6.8

[99] Grott, N., and Rovelli, C., "Weave states in loop quantum gravity", Gen. Relativ. Gravit., To appear, (1997). 7.2, 10

[100] Guichardet, A., Lecture Notes in Mathematics N. 261, (Springer-Verlag, Berlin, 1972). 6.8

[101] Hartle, J., "The Quantum Mechanics of Cosmology", in Coleman, S., Hartle, J., Piran, T., and Weinberg, S., eds., Quantum Cosmology and Baby Universes, (World Scientific, Singapore, 1991). 5.4

[102] Hartle, J., "Spacetime Quantum Mechanics and the Quantum Mechanics of Spacetime", in Julia, B., and J., Zinn, eds., Gravitation et Quantification. Les Houches, Session LVII 1992, (Elsevier Science, Amsterdam, 1995). 5.4, 9

[103] Hartle, J., and Hawking, S.W., "Wave function of the universe", Phys. Rev. D, 28, 2960-2975, (1983). 2

[104] Hawking, S., "Black hole explosion", Nature, 248, 30-31, (1974). 7.2

[105] Hawking, S., "Particle creation by black hole", Commun. Math. Phys., 43, 190-220, (1975). 7.2

[106] Hawking, S.W., "Quantum Cosmology", in DeWitt, B., and Stora, R., eds., Relativity, Groups and Topology, Les Houches Session XL, 333-379, (North Holland, Amsterdam, 1984). 2

[107] Higuchi, A., "Linearized gravity in DeSitter spacetime as a representation of SO(4,1)", Class. Quantum Grav., 8, 2005-2021, (1991). 6.8

[108] Horowitz, G., Lowe, D.A., and Maldcena, J., "Statistical Entropy of Nonextremal Four-Dimensional Black Holes and U-Duality", Phys. Rev. Lett., 77, 430-433, (1996). For a related online version see: G. Horowitz, et al., "Statistical Entropy of Nonextremal Four-Dimensional Black Holes and U-Duality", (February, 1996), [Online Los Alamos Preprint Archive]: cited on 29 September 1997, http://xxx.lanl.gov/abs/ hep-th/9603195. 2.3

[109] Horowitz, G., Maldacena, J., and Strominger, A., "Nonextremal Black Hole Microstates and U-duality", Phys. Lett. B, 383, 151-159, (1996). For a related online version see: G. Horowitz, et al., "Nonextremal Black Hole Microstates and U-duality", (March, 1996), [Online Los Alamos Preprint Archive]: cited on 29 September 1997, http://xxx.lanl.gov/ abs/hep-th/9603109. 2.3 
[110] Horowitz, G., and Strominger, A., "Counting States of Near-Extremal Black Holes", Phys. Rev. Lett., 77, 2368-2371, (1996). For a related online version see: G. Horowitz, et al., "Counting States of Near-Extremal Black Holes", (February, 1996), [Online Los Alamos Preprint Archive]: cited on 29 September 1997, http://xxx.lanl.gov/abs/hep-th/9602051. 2.3

[111] Husain, V., "Intersecting loop solutions of the hamiltonian constraint of quantum general relativity", Nulc. Phys. B, 313(3), 711-724, (1988). 3

[112] Husain, V., "Intersecting loop solutions of the Hamiltonian constraint of quantum general relativity", Nulc. Phys. B, 313, 711-724, (1989). 7.1

[113] Immirzi, G., "Real and complex connections for canonical gravity", (December, 1996), [Online Los Alamos Preprint Archive]: cited on 29 September 1997, http://xxx.lanl.gov/abs/gr-qc/9612030. 6

[114] Immirzi, G., "Quantum gravity and Regge calculus", (January, 1997), [Online Los Alamos Preprint Archive]: cited on 29 September 1997, http: //xxx.lanl.gov/abs/gr-qc/9701052. 6

[115] Immirzi, G., "Quantizing Regge Calculus", Class. Quantum Grav., 13, 2385-2394, (1996). For a related online version see: G. Immirzi, "Quantizing Regge Calculus", (December, 1995), [Online Los Alamos Preprint Archive]: cited on 29 September 1997, http://xxx.lanl.gov/abs/ gr-qc/9512040. 6

[116] Imperial College, "Theoretical Physics Group Home page", (1997), [Online HTML Document]: cited on 29 September 1997, http://euclid. tp.ph.ic.ac.uk/. 目

[117] Isham, C.J., "Topological and global aspects of quantum theory", in DeWitt, B., and Stora, R., eds., Relativity, Groups and Topology, Les Houches Session XL, 1059-1290, (North Holland, Amsterdam, 1984). 5.1, 田

[118] Isham, C.J., "Quantum Logic and the Histories Approach to Quantum Theory", J. Math. Phys., 35, 2157-2185, (1994). 5.4, 9

[119] Isham, C.J., "Quantum Logic and Decohering Histories", in Tchrakian, D.H., ed., Topics in Quantum Field Theory, (World Scientific, Singapore, 1995). 5.4, 9

[120] Isham, C.J., "Structural Problems Facing Quantum Gravity Theory", in Francaviglia, M., Longhi, G., Lusanna, L., and Sorace, E., eds., Proceedings of the 14th International Conference on General Relativity and Gravitation, 167-209, (World Scientific, Singapore, 1997). For a related online version see: C.J. Isham, "Structural Problems Facing Quantum Gravity Theory", (October, 1995), [Online Los Alamos Preprint Archive]: cited on 29 September 1997, http://xxx.lanl.gov/abs/gr-qc/9510065. 2

Living Reviews in Relativity (1998-1)

http://www.livingreviews.org 
[121] Isham, C.J., and Linde, N., "The classification of decoherence functionals: An analogue of Gleason's theorem", J. Math. Phys., 35, 6300-6370, (1994). 5.4, 9

[122] Isham, C.J., and Linde, N., "Quantum temporal logic and decoherent functionals in the histories approach to generalized quantum theory", $J$. Math. Phys., 35, 5452-5476, (1994). 5.4, 9

[123] Iwasaki, J., "A definition of the Ponzano-Regge quantum gravity model in terms of surfaces", J. Math. Phys., 36(11), 6288-6298, (1995). 3, 6.10

[124] Iwasaki, J., and Rovelli, C., "Gravitons as Embroidery on the Weave", Int. J. Mod. Phys. D, 1(3/4), 533-557, (1992). 3, 7.2, 10

[125] Iwasaki, J., and Rovelli, C., "Gravitons from loops: non-perturbative loop-space quantum gravity contains the graviton-physics approximation", Class. Quantum Grav., 1, 1653-1656, (1994). 10

[126] Jacobson, T., Seminar at the Schrödinger Institute, Vienna, (1996). 7.2

[127] Jacobson, T., and Smolin, L., "Nonperturbative quantum geometries", Nucl. Phys. B, 299(2), 295-345, (1988). 3

[128] Kauffman, L.H., "Vassiliev Invariants and the Loop States in Quantum Gravity", in Baez, J.C., ed., Knots and Quantum Gravity, 77-96, (Oxford U. Press, Oxford, 1994). 7.1

[129] Kauffman, L.H., and Lins, S.L., Temperley-Lieb Recoupling Theory and Invariant of 3-Manifolds, (Princeton University Press, Princeton, 1994). 6.7, 6.7

[130] Kodama, H., "Holomorphic wave function of the universe", Phys. Rev. D, 42, 2548-2565, (1990). 3, 7.1

[131] Krasnov, K., "Quantum loop representation for fermions coupled to Einstein-Maxwell field", Phys. Rev. D, 53, 1874-1888, (1996). 3, 9

[132] Krasnov, K., "Quantum Loop Representation for Fermions coupled to Einstein-Maxwell field", Phys. Rev. D, 53(4), 1874-1888, (1996). For a related online version see: K. Krasnov, "Quantum Loop Representation for Fermions coupled to Einstein-Maxwell field", (June, 1995), [Online Los Alamos Preprint Archive]: cited on 29 September 1997, http:// xxx.lanl.gov/abs/gr-qc/9506029. 7.1

[133] Krasnov, K., "Geometrical entropy from loop quantum gravity", Phys. Rev. D, 55(6), 3505-3513, (1997). For a related online version see: K. Krasnov, "Counting Surface States in the loop quantum gravity", (March, 1996), [Online Los Alamos Preprint Archive]: cited on 29 September 1997, http://xxx.lanl.gov/abs/gr-qc/9603025. [3, 6, 7.2 
[134] Krasnov, K., "On statistical mechanics of Schwarzschild black hole", Gen. Relativ. Gravit, in print, (1997). For a related online version see: K. Krasnov, "On statistical mechanics of gravitational systems", (May, 1996), [Online Los Alamos Preprint Archive]: cited on 29 September 1997, http://xxx.lanl.gov/abs/gr-qc/9605047. [3, 6, , 7.2

[135] Kuchař, K., Recalled from memory from a discussion at the conference "Quantum Gravity in the Southern Cone" in Montevideo, (1996). 3

[136] Lewandowski, J., "Topological Measure and Graph-Differential Geometry on the Quotient Space of Connections", Int. J. Mod. Phys. D, 3, 207-210, (1994). For a related online version see: J. Lewandowski, "Topological Measure and Graph-Differential Geometry on the Quotient Space of Connections", (June, 1994), [Online Los Alamos Preprint Archive]: cited on 29 September 1997, http://xxx.lanl.gov/abs/gr-qc/9406025. 3, 6.2

[137] Lewandowski, J., "The Operators of Quantum Gravity", Lecture given at the Workshop on Canonical Quantum Gravity, Warsaw, (1995). 3, 6.5

[138] Lewandowski, J., "Volume and Quantizations", Class. Quantum Grav., 14, 71-76, (1997). For a related online version see: J. Lewandowski, "Volume and Quantizations", (February, 1996), [Online Los Alamos Preprint Archive]: cited on 29 September 1997, http://xxx.lanl.gov/abs/ gr-qc/9602035. 3, 6.6, 7.2, 8

[139] Lewandowski, J., and Marolf, D., "Loop constraints: A habitat and their algebra", (1997), [Online Los Alamos Preprint Archive]: cited on 29 September 1997, http://xxx.lanl.gov/abs/gr-qc/? 3, 3, 8, 9]

[140] Loll, R., "Nonperturbative solutions for lattice quantum gravity", Nucl. Phys. B, 444, 619-639, (1995). 3, 7.1

[141] Loll, R., "The volume operator in discretized quantum gravity", Phys. Rev. Lett., 75, 3048-3051, (1995). For a related online version see: R. Loll, "The volume operator in discretized quantum gravity", (June, 1995), [Online Los Alamos Preprint Archive]: cited on 29 September 1997, http://xxx.lanl.gov/abs/gr-qc/9506014. 3, 7.2

[142] Loll, R., "Spectrum of the Volume Operator in Quantum Gravity", Nucl. Phys. B, 460(1), 143-154, (1996). For a related online version see: R. Loll, "Spectrum of the Volume Operator in Quantum Gravity", (November, 1995), [Online Los Alamos Preprint Archive]: cited on 29 September 1997, http://xxx.lanl.gov/abs/gr-qc/9511030. [3, 7.2

[143] Markopoulou, F., and Smolin, L., "Causal evolution of spin networks", (February, 1997), [Online Los Alamos Preprint Archive]: cited on 29 September 1997, http://xxx.lanl.gov/abs/gr-qc/9702025. 3, 6.10, प]

Living Reviews in Relativity (1998-1)

http://www.livingreviews.org 
[144] Marolf, D., Green's bracket algebra and their quantization, PhD Thesis, (University of Texas at Austin, Austin, Texas, 1992). 3, 6.8

[145] Marolf, D., "Loop Representation for 2+1 Gravity on a Torus", Class. Quantum Grav., 10, 2625-2648, (1993). For a related online version see: D. Marolf, "Loop Representation for 2+1 Gravity on a Torus", (March, 1993), [Online Los Alamos Preprint Archive]: cited on 29 September 1997, http://xxx.lanl.gov/abs/gr-qc/9303019. 3, 7.1

[146] Marolf, D., "Quantum Observables and Recollapsing Dynamics", Class. Quantum Grav., 12, 1199-1220, (1995). 3, 6.8

[147] Marolf, D., "The spectral analysis inner product", in Ruffini, R., and Keiser, M., eds., Proceedings of the VIIth Marcel Grossman Conference, (World Scientific, Singapore, 1995). 3, 6.8

[148] Marolf, D., and Mourão, J.M., "On the support of the AshtekarLewandowski measure", Commun. Math. Phys., 170, 583-605, (1995). For a related online version see: D. Marolf, et al., "On the support of the Ashtekar-Lewandowski measure", (March, 1994), [Online Los Alamos Preprint Archive]: cited on 29 September 1997, http://xxx.lanl.gov/ abs/hep-th/9403112. 3, 6.7

[149] Morales-Técotl, H.A., and Rovelli, C., "Fermions in quantum gravity", Phys. Rev. Lett., 72, 3642-3645, (1994). For a related online version see: H.A. Morales-Técotl, et al., "Fermions in quantum gravity", (January, 1994), [Online Los Alamos Preprint Archive]: cited on 29 September 1997, http://xxx.lanl.gov/abs/gr-qc/9401011. 3, 7.1, 9

[150] Morales-Técotl, H.A., and Rovelli, C., "Loop Space Representation of Quantum Fermions and Gravity", Nucl. Phys. B, 451, 325-361, (1995). 3], [.1, 9

[151] Penrose, R., "Angular momentum: an approach to combinatorial spacetime", in Bastin, T., ed., Quantum Theory and Beyond, 151-180, (Cambridge University Press, Cambridge, 1971). 3, 6.3

[152] Penrose, R., "Applications of negative dimensional tensors", in Welsh, D., ed., Combinatorial Mathematics and its Application, 221-243, (Acad. Press, London, 1971). 3, 6.3

[153] Penrose, R., "The twistors program", Rep. Math. Phys., 12, 65-76, (1977). 】

[154] Penrose, R., The Emperor's new Mind, (Oxford University Press, Oxford, 1989). 5.4

[155] Pittsburgh Relativity Group, "Pittsburgh Relativity Group", (1997), [Online HTML Document]: cited on 29 September 1997, http://artemis. phyast.pitt.edu/. 团 
[156] Pullin, J., "Center for Gravity and Geometry", (1997), [Online HTML Document]: cited on 29 September 1997, http://vishnu.nirvana. phys.psu.edu. 团

[157] Pullin, J., "Knot invariants as nondegenerate states of four-dimensional quantum gravity", in Lucio, J., ed., Proceedings of the Vth Mexican School of Particles and Fields, (World Scientific, Singapore, 1993). 3, 7.1]

[158] Reisenberger, M., "Worldsheet formulations of gauge theories and gravity", (December, 1994), [Online Los Alamos Preprint Archive]: cited on 29 September 1997, http://xxx.lanl.gov/abs/gr-qc/9412035. 3, 6.10

[159] Reisenberger, M., "A Left-Handed Simplicial Action for Euclidean General Relativity", Class. Quantum Grav., 14, 1730-1770, (1997). For a related online version see: M. Reisenberger, "A Left-Handed Simplicial Action for Euclidean General Relativity", (September, 1996), [Online Los Alamos Preprint Archive]: cited on 29 September 1997, http://xxx.lanl.gov/ abs/gr-qc/9609002. 3, 3, 7.1

[160] Reisenberger, M., and Rovelli, C., "Sum over Surfaces form of Loop Quantum Gravity", Phys. Rev. D, 56, 3490-3508, (1997). For a related online version see: M. Reisenberger, et al., "Sum over Surfaces form of Loop Quantum Gravity", (December, 1996), [Online Los Alamos Preprint Archive]: cited on 29 September 1997, http://xxx.lanl.gov/abs/ gr-qc/9612035. 3, 3, 6.10, 6.10, 8, 9, 9, 10

[161] Rovelli, C., "2nd Warsaw Workshop on Canonical and Quantum Gravity, in Matters of Gravity, J. Pullin ed.", (September, 1997), [Online Los Alamos Preprint Archive]: cited on 29 September 1997, http: //xxx.lanl.gov/abs/gr-qc/9709023. 圈

[162] Rovelli, C., On the Quantization of the Theory of Gravity, PhD Thesis, (Università di Trento, Trento, Italy, 1987). 6.8

[163] Rovelli, C., "The Loop Space Representation of Quantum General Relativity", in Ashtekar, A., ed., New perspectives in canonical gravity, (Bibliopolis, Naples, 1989). 3

[164] Rovelli, C., "Ashtekar's formulation of general relativity and loop-space non-perturbative quantum gravity : a report", Class. Quantum Grav., 8(9), 1613-1675, (1991). 田, 6

[165] Rovelli, C., "Quantum evolving constants", Phys. Rev. D, 44(4), 13391341, (1991). 5.4

[166] Rovelli, C., "Quantum mechanics without time: a model", Phys. Rev. D, 42(8), 2638-2646, (1991). 5.4

Living Reviews in Relativity (1998-1)

http://www.livingreviews.org 
[167] Rovelli, C., "Quantum reference systems", Class. Quantum Grav., 8(2), 317-331, (1991). 9

[168] Rovelli, C., "Time in quantum gravity: an hypothesis", Phys. Rev. D, 43(2), 442-456, (1991). 5.4, 7.1

[169] Rovelli, C., "What is observable in classical and quantum gravity?", Class. Quantum Grav., 8(2), 297-316, (1991). 5.3, 9

[170] Rovelli, C., "Area is the length of Ashtekar's triad field", Phys. Rev. D, 47, 1703-1705, (1993). 8

[171] Rovelli, C., "Basis of the Ponzano-Regge-Turaev-Viro-Ooguri quantumgravity model is the loop representation basis", Phys. Rev. D, 48, 27021707, (1993). 6.10

[172] Rovelli, C., "A generally covariant quantum field theory and a prediction on quantum measurements of geometry", Nulc. Phys. B, 405, 797-815, (1993). 7.2, 8

[173] Rovelli, C., "Statistical mechanics of gravity and thermodynamical origin of time", Class. Quantum Grav., 10(8), 1549-1566, (1993). 10

[174] Rovelli, C., "The statistical state of the universe", Class. Quantum Grav., 10(8), 1567-1578, (1993). 10

[175] Rovelli, C., "Outline of a generally covariant quantum field theory and a quantum theory of gravity", J. Math. Phys., 36, 6529-6547, (1995). For a related online version see: C. Rovelli, "Outline of a generally covariant quantum field theory and a quantum theory of gravity", (March, 1995), [Online Los Alamos Preprint Archive]: cited on 29 September 1997, http: 7/xxx.lanl.gov/abs/gr-qc/9503067. 6.9, 7.1, 10

[176] Rovelli, C., "Black Hole Entropy from Loop Quantum Gravity", Phys. Rev. Lett., 14, 3288-3291, (1996). For a related online version see: C. Rovelli, "Black Hole Entropy from Loop Quantum Gravity", (March, 1996), [Online Los Alamos Preprint Archive]: cited on 29 September 1997, http://xxx.lanl.gov/abs/gr-qc/9603063. [3, 6, 7.2

[177] Rovelli, C., "Localization in quantum field theory, which aspects or quantum field theory are compatible with what we know about spacetime?", in Cao, J., ed., Proceedings of the 1996 Boston Colloquium for Philosophy of Science, Reflections on the Foundations of Quantum Field theory, (Boston Center for the Philosophy of Science, Boston, 1996). 5.3

[178] Rovelli, C., "Loop Quantum Gravity and Black hole Physics", Helv. Phys. Acta, 69, 582-611, (1996). For a related online version see: C. Rovelli, "Loop Quantum Gravity and Black hole Physics", (August, 1996), [Online Los Alamos Preprint Archive]: cited on 29 September 1997, http://xxx. lanl.gov/abs/gr-qc/9608032. [6, 10 
[179] Rovelli, C., "Relational Quantum Mechanics", Int. J. Theor. Phys., 35(8), 1637-1678, (1996). For a related online version see: C. Rovelli, "Relational Quantum Mechanics", (September, 1996), [Online Los Alamos Preprint Archive]: cited on 29 September 1997, http://xxx.lanl.gov/ abs/quant-ph/9609002. 5.4

[180] Rovelli, C., "Half way through the woods", in Earman, J., and Norton, J., eds., The Cosmos of Science, 180-223, (University of Pittsburgh Press and Universitäts Verlag Konstanz, Konstanz, 1997). 2, 5.3, 5.4

[181] Rovelli, C., "Quantum Gravity as a Sum over Surfaces", Nulc. Phys. B, (Proc. Suppl.) 57, 28-43, (1997). 3, 6.10, 9

[182] Rovelli, C., and Smolin, L., "A new approach to quantum gravity based on loop variables", International conference on Gravitation and Cosmology, Goa, Dec 14-19 India, (1987). 1, 3

[183] Rovelli, C., and Smolin, L., "Knot theory and quantum gravity", Phys. Rev. Lett., 61, 1155-1158, (1988). 3, 6.1, 7.1

[184] Rovelli, C., and Smolin, L., "Loop space representation of quantum gen-

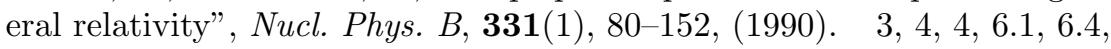
6.6, [.6, 6.7, 7.1$]$

[185] Rovelli, C., and Smolin, L., "The physical Hamiltonian in nonperturbative quantum gravity", Phys. Rev. Lett., 72, 446-449, (1994). 3

[186] Rovelli, C., and Smolin, L., "Discreteness of area and volume in quantum gravity", Nucl. Phys. B, 442, 593-622, (1995). For a related online version see: C. Rovelli, et al., "Discreteness of area and volume in quantum gravity", (November, 1994), [Online Los Alamos Preprint Archive]: cited on 29 September 1997, http://xxx.lanl.gov/abs/gr-qc/9411005. Erratum: Nucl. Phys., B456, 734, (1995). 3, 6.5, 7.2, 7.2, 7.2, 8

[187] Rovelli, C., and Smolin, L., "Spin Networks and Quantum Gravity", Phys. Rev. D, 52, 5743-5759, (1995). For a related online version see: C. Rovelli, et al., "Spin Networks and Quantum Gravity", (May, 1995), [Online Los Alamos Preprint Archive]: cited on 29 September 1997, http://xxx. lanl.gov/abs/gr-qc/9505006. [3, 6.3, 6.4

[188] Rovelli, C., and Smolin, L., "The physical hamiltonian in nonperturbative quantum gravity", Phys. Rev. Lett., 72(4), 1994, (446-449). For a related online version see: C. Rovelli, et al., "The physical hamiltonian in nonperturbative quantum gravity", (August, 1993), [Online Los Alamos Preprint Archive]: cited on 29 September 1997, http: 7/xxx.lanl.gov/abs/gr-qc/9308002. 6.9, 7.1

[189] Rovelli, C., and Thiemann, T., "The Immirzi parameter in quantum general relativity", (May, 1997), [Online Los Alamos Preprint Archive]: cited

Living Reviews in Relativity (1998-1)

http://www.livingreviews.org 
on 29 September 1997, http://xxx.lanl.gov/abs/gr-qc/9705059. 6), प.2

[190] Sen, A., "Gravity as a spin system", Phys. Lett. B, 119, 89-91, (1982). [3, [6, 6]

[191] Smolin, L., "Loops and Strings", (1998), [Article in the Online Journal Living Reviews in Relativity]: cited on 29 September 1997. To appear. [2.3]

[192] Smolin, L., "The classical limit and the form of the hamiltonian constraint in nonperturbative quantum general relativity", (September, 1996), [Online Los Alamos Preprint Archive]: cited on 29 September 1997, http: //xxx.lanl.gov/abs/gr-qc/9609034. B, 8, 9

[193] Smolin, L., "Macroscopic deviations from Hawking radiation?", (February, 1996), [Online Los Alamos Preprint Archive]: cited on 29 September 1997, http://xxx.lanl.gov/abs/gr-qc/9602001. 7.2

[194] Smolin, L., "The Bekenstein Bound, Topological Quantum Field Theory and Pluralistic Quantum Field Theory", (August, 1995), [Online Los Alamos Preprint Archive]: cited on 29 September 1997, http: //xxx.lanl.gov/abs/gr-qc/9508064. 7.2

[195] Smolin, L., "Knot Theory in Quatum Gravity", in Ashtekar, A., ed., New Perspectives in canonical gravity, (Bibliopolis, Naples, 1988). 3

[196] Smolin, L., "Recent developments in nonperturbative quantum gravity", in Perez-Mercader, J., Sola, J., and Verdaguer, E., eds., Proceedings of the XXII Gift International Seminar on Theoretical Physics, Quantum Gravity and Cosmology, June 1991, Catalonia, Spain, (World Scientific, Singapore, 1992). 3, 6.5

[197] Smolin, L., The Life of the Cosmos, (Oxford University Press, Oxford, 1997). 2

[198] Strominger, A., and Vafa, G., "Microscopic Origin of the BekensteinHawking Entropy", Phys. Lett. B, 379, 99-104, (1996). For a related online version see: A. Strominger, et al., "Microscopic Origin of the Bekenstein-Hawking Entropy", (January, 1996), [Online Los Alamos Preprint Archive]: cited on 29 September 1997, http://xxx.lanl.gov/ abs/hep-th/9601029. 2.3

[199] Syracuse University, "Relativity Group", (1997), [Online HTML Document]: cited on 29 September 1997, http://www.phy.syr.edu/ research/relativity/. [t

[200] 't Hooft, G., "Can spacetime be probed below the string size?", Phys. Lett., 198, 61-63, (1987). 2.3 
[201] Thiemann, T., "QSD V : Quantum Gravity as the Natural Regulator of Matter Quantum Field Theories", (May, 1997), [Online Los Alamos Preprint Archive]: cited on 29 September 1997, http://xxx.lanl.gov/ abs/gr-qc/9705019. 3, 7.1, 9]

[202] Thiemann, T., "Quantum Spin Dynamics (QSD)", (June, 1996), [Online Los Alamos Preprint Archive]: cited on 29 September 1997, http://xxx. lanl.gov/abs/gr-qc/9606089. 3, 6, 6.9, 7.1, 8

[203] Thiemann, T., "Quantum Spin Dynamics (QSD) II", (June, 1996), [Online Los Alamos Preprint Archive]: cited on 29 September 1997, http://xxx. lanl.gov/abs/gr-qc/9606090. [3, 6, 6.9, 7.1, 8

[204] Thiemann, T., "Kinematical Hilbert Spaces for Fermionic and Higgs Quantum Field Theories", (May, 1997), [Online Los Alamos Preprint Archive]: cited on 29 September 1997, http://xxx.lanl.gov/abs/ gr-qc/9705021. 7.1, 9

[205] Thiemann, T., "Closed formula for the matrix elements of the volume operator in canonical quantum gravity", (June, 1991), [Online Los Alamos Preprint Archive]: cited on 29 September 1997, http://xxx.lanl.gov/ abs/gr-qc/9606091. 7.2, 7.2

[206] Thiemann, T., "A length operator for canonical quantum gravity", (June, 1996), [Online Los Alamos Preprint Archive]: cited on 29 September 1997, http://xxx.lanl.gov/abs/gr-qc/9606092. 7.2

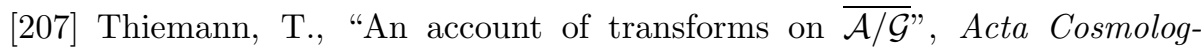
ica, 21(2), 145-167, (1996). For a related online version see: T. Thiemann, "An account of transforms on $\overline{\mathcal{A} / \mathcal{G}}$ ", (November, 1995), [Online Los Alamos Preprint Archive]: cited on 29 September 1997, http: //xxx.lanl.gov/abs/gr-qc/9511049. 3

[208] Thiemann, T., "Anomaly-Free Formulation of Nonperturbative Fourdimensional Lorentzian Quantum Gravity", Phys. Lett., 380, 257-264, (1996). For a related online version see: T. Thiemann, "Anomaly-Free Formulation of Nonperturbative Four-dimensional Lorentzian Quantum Gravity", (June, 1996), [Online Los Alamos Preprint Archive]: cited on 29 September 1997, http://xxx.lanl.gov/abs/gr-qc/9606088. [3, 6, 6.9, [7.1, 8

[209] Verlinde, H., and Verlinde, E., "Scattering at Planckian energies", Nucl. Phys. B, 371, 246-268, (1992). 2.3

[210] Wald, R.M., Quantum Field Theory on Curved Spacetime and Black Hole Thermodynamics, (University of Press, Chicago, 1994). 7.2

[211] Wilson, K., and Kogut, "Hamiltonian Formulation Of Wilson's Lattice Gauge Theories", Phys. Rev. D, 11, 395-408, (1975). 3

Living Reviews in Relativity (1998-1)

http://www.livingreviews.org 
[212] Witten, E., "Quantum Field Theory And The Jones Polynomial", Commun. Math. Phys., 121(3), 351-399, (1989). 7.1

[213] York, J.W., "Dynamical origin of black hole radiance", Phys. Rev. D, 28(12), 2929-2945, (1983). 7.2

[214] Yutsin, A.P., Levinson, J.B., and Vanagas, V.V., Mathematical Apparatus of the Theory of Angular Momentum, (Israel program for Scientific Translation, Jerusalem, 1962). 6.4

[215] Zapata, J.A., "A combinatorial approach to diffeomorphism invariant quantum gauge theories", (March, 1997), [Online Los Alamos Preprint Archive]: cited on 29 September 1997, http://xxx.lanl.gov/abs/ gr-qc/9703037. 6.8

[216] Zapata, J.A., "Combinatorial space from loop quantum gravity", (March, 1997), [Online Los Alamos Preprint Archive]: cited on 29 September 1997, http://xxx.lanl.gov/abs/gr-qc/9703038. 6.8

[217] Zegwaard, J., "The weaving of curved geometries", Phys. Lett., 300, 217222, (1993). 7.2 